\title{
Multi-physics problems computation using numerically adapted meshes: application to magneto-thermo-mechanical systems
}

\author{
Antoine Alexandre Journeaux ${ }^{1, a}$, Frédéric Bouillault ${ }^{1}$, and Jean-Yves Roger ${ }^{2}$ \\ ${ }^{1}$ Laboratoire de Génie Électrique de Paris (UMR 8507), 11 rue Joliot Curie, Plateau du Moulon, 91192 Gif-sur-Yvette, France \\ ${ }^{2}$ EDF R\&D, 1 avenue du Général De Gaulle, BP 408, 92141 Clamart, France
}

Received: 26 October 2012 / Accepted: 22 February 2013

Published online: 2 April 2013 - (c) The Author(s) 2013

\begin{abstract}
In physical systems, interactions between phenomena of different nature, generally coupled to each other, are often involved. Their comprehensive study requires the use of various physical models sharing a unique set of physical quantities. In an effort to correctly model these systems, numerical methods are frequently used. However, computational tools dedicated to such a specific scope of use are barely available. Furthermore, unsuited numerical discretization and high memory costs are two major drawbacks limiting the use of coupled numerical models. We present in this paper a global method which enables the independent use of existing computational tools, the numerical adaption to each physical model and the reduction of the memory use. This method has resorted to a unique discretization and topology for each physical model. The link between these independent models is ensured by the projection of quantities common to them. Thus computational tools, originally not intended to operate together, can be used again. After a theoretical description of the projection method, we will present successive application to discretizations of different nature. Thus, numerical efficiency of the projections in themselves will be tested. Because of the large range of combination of physical models, additional tests will be carried out in order to determine the most accurate coupling flowchart. A highly coupled problem, involving three different physical models, will be presented using the projection method. Results show a significant gain in flexibility and cuts in memory costs. Present test-cases reveal that accuracy is of same order as the one obtained using dedicated tools.
\end{abstract}

\section{Introduction}

Advanced studies of physical phenomena often require the use of numerical methods. These computational tools are of great help as they can be used to reveal immeasurable values or improve the resolution of experimental data. Another major use consists in the prediction or the optimization of a system before its implementation.

The bases for the computation of a physical phenomenon are physical models and numerical approximations. The physical part of the study is usually well determined and generally involves partial differential equations governing the physics (e.g., Navier-Stokes equations, heat law, Maxwell equations and so on). If these physical laws are human readable, they cannot be used in that form for numerical computing. The numerical part of such a study focuses on the processing of these equations, which turn the physical laws, into a computational form. It generally invokes an approximation of the physical fields (as shown in Fig. 1) where differential equations are replaced by algebraic systems. The numerical approximation is based on the decomposition of the physical domain into elementary

\footnotetext{
a e-mail: journeaux@lgep.supelec.fr
}

blocks in which physical fields have predefined shape. This operation is called the discretization process (Fig. 1b) and uses meshed version of the geometry.

Obviously the mesh depends on the geometry of the system, while the choice of the "predefined shape" - called basis functions or discretization functions - depends on (a)

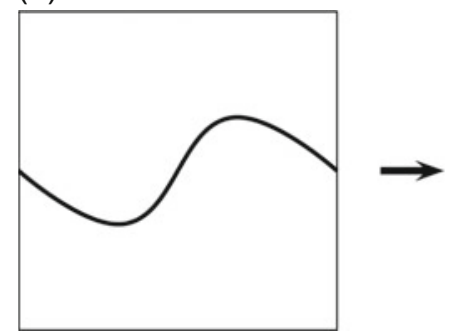

(b)

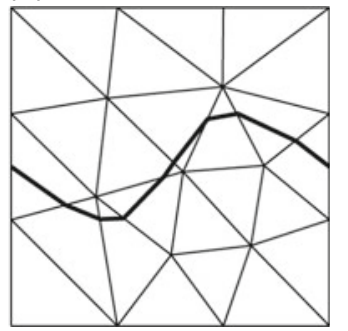

Fig. 1. Example of numerical discretization of a physical problem. (a) Symbolizes the continuous problem, or exact problem. (b) The numerical counterpart which is computable with numerical tools. Emphasized solid lines symbolize the exact solution and its discrete counterpart. 


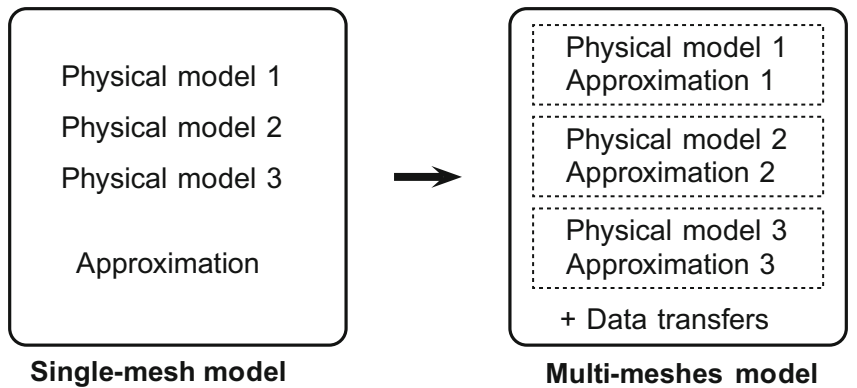

Fig. 2. Sketch of the method: rather than developing a specific numerical program for the coupled problem, the numerical model is decomposed into sub-problems for which heterogeneous numerical process are applied.

the physical model itself. Consequently the way the mesh, and more generally the numerical model, is built will depend on the physical one.

Accurate design of electromagnetic devices often involves multiple physical models. These models are generally linked to each other and require particular treatment. Numerical implementations usually have resorted to dedicated tools which solve the problems through a unique process [1]. Developing a program for each class of problems is often time consuming and hardens the software servicing. Moreover, each part of code might not fit the needs for other applications. The use of a unique mesh, another major drawback, prevents the numerical adaptation of all physical models simultaneously. At the same time, these methods frequently present a high memory cost.

The present work introduces an innovative method which enables the flexible computation of coupled problems using existing computational tools, the numerical adaptation to each physical model and the reduction of the computational cost.

Instead of attempting to solve the problem in a single computation, the entire process can be wisely decomposed into individual sub-problems (see Fig. 2) [2]. Data handling is easier for one sub-problem and it allows the use of numerically adapted codes, thus improving the global accuracy. This method also reduces the memory cost as the meshes are topologically adapted to the physical geometry. In addition, it allows the effortless reuse of existing computer programs, and the replacement of sub-codes or sub-problems is easy. The computational overcost is not prohibitive compared to the classical processing, and advantageous features are introduced.

In the present method data transfer and iterative processes are used to link the different sub-problems to each other. The focus of this article is on the data transfer stage - which is the most complex part of the process - and its use in multi-physics problems.

In order to achieve an accurate coupling process, data transfer methods will be intensively tested in the first part. Once the most appropriate method is determined, coupling values and iterative processes will be considered in the second part. The aim of this work is to present a set of operations that computes coupled problems as accurately as possible. The robustness will be measured using several analytical tests. Validation cases based on magnetothermo-mechanical coupling will then be presented.

\section{Theoretical aspects}

\subsection{Magnetic model}

For the sake of simplicity we first consider static magnetic formulations. We consequently assume that motion induces negligible currents through the system. Eddy currents are considered as negligible when they are low enough compared to the supplied ones, or when their associated magnetic fields have negligible influence. Nevertheless, the study of transient magnetic systems can be performed with ease using the same technique.

\subsubsection{Steady-state Maxwell equations}

As we consider static formulations the calculation of magnetic and electric fields is decoupled. Displacement currents are also neglected, Maxwell equations reduce to the Ampere's and Gauss's laws for magnetism:

$$
\begin{aligned}
\operatorname{curl} \mathbf{H} & =\mathbf{J}_{g} \\
\operatorname{div} \mathbf{B} & =0 .
\end{aligned}
$$

$\mathbf{B}, \mathbf{H}$ and $\mathbf{J}_{g}$ respectively denote the magnetic flux density, the magnetic field and the source current density. They are vector fields of the three-dimensional space. The linear constitutive law, $\mathbf{B}=\mu \mathbf{H}$ where $\mu$ is the magnetic permeability, establishes the relation between $\mathbf{B}$ and $\mathbf{H}$. Previous equations lead to a problem which can be rewritten with either scalar or vector potentials. These two equivalent versions of the problem are respectively called the "electric formulation" and the "magnetic formulation".

\subsubsection{Electric formulation}

Because the magnetic computational domain $\left(\mathcal{D}_{\text {mag }}\right)$ is assumed to be star-shaped, for a locally $\mathcal{C}^{1}$ smoothness of the magnetic induction and assuming $\operatorname{div} \mathbf{B}$ is null, the Poincaré lemma can be applied. There then exists a smooth field A such that:

$$
\mathbf{B}=\operatorname{curl} \mathbf{A},
$$

where $\mathbf{A}$ is the so-called magnetic vector potential. Assuming $\operatorname{div} \mathbf{B}=0$ and replacing $\mathbf{H}$ in the Ampere's law one obtains:

$$
\operatorname{curl}\left(\frac{1}{\mu} \operatorname{curl} \mathbf{A}\right)=\mathbf{J}_{g} .
$$

This equation is solved using the weighted residual method and the boundary condition $\mathbf{A} \times n=0$. This ensures that $\mathbf{B} \times n=0$ where $n$ is the local normal to boundary. 
The weak form of the previous equation - where the boundary integral is zero - is:

$$
\int_{\mathcal{D}_{m a g}} \frac{1}{\mu} \operatorname{curl} \text { Acurl } \psi=\int_{\mathcal{D}_{m a g}} \mathbf{J}_{g} \psi,
$$

where $\psi$ is a test function belonging to the same sub-space as $\mathbf{A}$. The discretization of the problem leads to a linear equation of the form: $[P][A]=\left[J_{g}\right]$.

\subsubsection{Magnetic formulation}

Under the same assumption on the computational domain as in Section 1.1.2, the Poincaré lemma also applies to the $\mathbf{H}-\mathbf{H}_{g}$ field which has a zero curl. A scalar field $\Omega$ exists and satisfies:

$$
\mathbf{H}=\mathbf{H}_{g}-\operatorname{grad} \Omega .
$$

$\mathbf{H}_{g}$ is an intermediate field deduced from the source current density and defined as $\mathbf{J}_{g}=\operatorname{curl} \mathbf{H}_{g} \cdot{ }^{1}$ Replacing $\mathbf{B}$ in Gauss's law leads to the magnetic formulation:

$$
\operatorname{div}(\mu \operatorname{grad} \Omega)=\operatorname{div}\left(\mu \mathbf{H}_{g}\right) .
$$

The drop of the boundary integral ensures the weak nullity of the normal component of the magnetic field. Applying the weighted residual method, one gets the so-called weak formulation:

$$
\int_{\mathcal{D}_{m a g}} \frac{1}{\mu} \operatorname{grad} \Omega \operatorname{grad} \psi=\int_{\mathcal{D}_{m a g}} \mathbf{H}_{g} \operatorname{grad} \psi .
$$

Once discretized, this formulation leads to a linear system of the form $[Q][\Omega]=\left[H_{g}\right]$.

Electric and magnetic formulations describe, in terms of potentials, the behavior of steady-state magnetic problems. Fields $\mathbf{B},\left(\mathbf{J}, \mathbf{J}_{g}\right), \mathbf{H}, \mathbf{A}$ and $\Omega$ are discretized using the Whitney complex [3]. The magnetic problem is solved with the help of code_Carmel3D. ${ }^{2}$

\subsection{Mechanical model}

Electrical systems often require to take into account parts which do not belong to the mechanical domain (e.g., air parts, static pieces or domains without interest). Thanks to the data transfer method, all of these parts can be excluded, which therefore significantly reduces the computational cost. This procedure will be applied to the linear elasticity problem.

Throughout a mechanical domain $\mathcal{D}_{m e c}$, for infinitesimal strains or "small" deformations, the equation of motion $^{3}$ is:

$$
\sigma_{i j, j}+f_{i}=\rho \partial_{t}^{2} u_{i}
$$

\footnotetext{
1 Precomputed using a spanning tree, analytical formula or finite element technique.

${ }^{2}$ code_Carmel3D is software codeveloped by EDF (French electricity company) and L2EP (Lille).

3 which is the local expression of the Newton's second law.
}

The previous equation is written using Einstein's notations and Cartesian coordinates. $\sigma$ represents the Cauchy stress tensor, $u$ is the displacement field and $\rho$ is the density. In the simplest case, stress and strain are related by:

$$
\sigma=\frac{E}{1+\nu}\left(\epsilon+\frac{\nu}{1-2 \nu} \operatorname{tr}(\epsilon) G\right)
$$

where $G$ is the metric tensor, ${ }^{4} E$ and $\nu$ are respectively the Young modulus and the Poisson's ratio, and $\operatorname{tr}($.$) is the$ trace operator. If the thermal problem is coupled with the mechanical one, the actual behavior law is more complex (see Sect. 1.4.3). Variations of the strain tensor can be described using the strain-displacement relation defined by:

$$
\epsilon_{i j}=\frac{1}{2}\left(u_{i, j}+u_{j, i}\right)
$$

Combining equations (8), (9) and (10) one gets the displacement formulation. Eliminating the strain as unknown, differentiating the stiffness tensor and finally substituting into the equilibrium equation, one obtains:

$$
\frac{E}{2(1+\nu)}\left(u_{i, j j}+\frac{\nu u_{i, i j}}{1-2 \nu}\right)+f_{i}=\rho \partial_{t}^{2} u_{i}
$$

Rewriting the formula in terms of energy functional and using the weighted residual method, in a compact way, leads to:

$$
\int_{\mathcal{D}_{m e c}} \rho \partial_{t}^{2} u \delta u+\int_{\mathcal{D}_{m e c}} \epsilon^{t} H \delta \epsilon-\int_{\mathcal{D}_{m e c}} f \delta u+\int_{\Gamma_{m e c}} F_{\Gamma} \partial u
$$

for all admissible displacement field $\delta u$. $H$ is a tensor which represents the Hooke law and $F$ is a surface load. $\delta \epsilon$ and $\epsilon$ are expressed in terms of $\delta u$ and $u$ using the strain-displacement relation (Eq. (10)).

Once discretized, the following linear system is obtained:

$$
[M] \partial_{t}^{2}[u]+[K][u]=[F] .
$$

It is solved thanks to the code_Aster ${ }^{5}$ tool with the help of quadratic interpolation functions.

The array $[F]$ is obtained using the electromagnetic force density, thus the determination of this field is required within the solely mechanical sub-problem (which may not cover the entire domain).

In mechanics, the use of quadratic interpolation functions is preferred as it enhances the solution accuracy in a very significant way. Not only because second-order functions are used, but also because strain is linear and parasitic values are avoided. This implies the use of adequate methods.

\footnotetext{
4 As we consider Cartesian coordinates, we always substitute $G$ by $I_{3}$.

${ }^{5}$ code_Aster is developed by EDF (the French electricity company).
} 


\subsection{Thermal model}

The distribution of the temperature as a function of the time is given by the thermal conduction problem where heat sources are Joule losses. Boundary heat fluxes were modeled using imposed heat fluxes which are proportional to the local temperature of the boundaries. As these losses were introduced, insofar as the Joule losses are constants, the system tends to produce a steady-state solution.

Considering a local energy balance (Eq. (14)) and the Fourier's conduction law for homogeneous materials (Eq. (15)), the thermal problem reduces to:

$$
\begin{aligned}
-\operatorname{div} \mathbf{q}+s & =\rho C_{p} \partial_{t} T, \\
\mathbf{q} & =-k \operatorname{grad} T .
\end{aligned}
$$

The heat equation rewrites in temperature $T$ as:

$$
k \Delta T+s=\rho C_{p} \partial_{t} T,
$$

where $\mathbf{q}$ is the vector of heat flux (directed along decreasing temperatures), $s$ is heat source density and $\rho C_{p}$ is the specific volume heat with constant temperature.

To model the thermal influence of external parts, heat losses are introduced - as boundary conditions - using the Newton's law of cooling. These conditions are thus imposed on the vector of heat flux:

$$
-\mathbf{q} \cdot \mathbf{n}=h\left(T-T_{e x t}\right),
$$

where $h$ is the local heat transfer coefficient, $\mathbf{n}$ is the outward unit normal and $T_{\text {ext }}$ is a constant temperature which represents the external domain. Equations (16) and (17) lead to the system (18) written in its weak form over a thermal domain $\mathcal{D}_{\text {the }}$.

$$
\begin{array}{r}
\int_{\Omega} \rho C_{p} \partial_{t} T \phi+\int_{\Omega} k \operatorname{grad} T \operatorname{grad} \phi+\int_{\Gamma} h T \phi \\
=\int_{\Omega} s \phi+\int_{\Gamma} h T_{\text {ext }} \phi .
\end{array}
$$

$\phi$ is a test function that vanishes at temperature imposed surfaces. It leads to the following linear system:

$$
[C] \partial_{t}[T]+[\Lambda][T]=\left[S_{\text {mag }}\right]+\left[H_{\text {ext }}\right] .
$$

This model is solved either by Code_Aster or code Syrthes. ${ }^{6}$

\subsection{Relationship between models}

Sub-models can interact in two different ways. The first interaction involves external loads resulting from the computation of another sub-model (e.g., the mechanical load which directly depends on magnetic unknowns). A second type of coupling is due to variations of physical properties.

\footnotetext{
${ }^{6}$ These codes were developed by EDF and are used for industrial thermal computations.
}

These variations generally depend on unknowns of other sub-problems (e.g., the mechanical behavior will depend on the temperature). These two kinds of interactions are taken into account in two distinct manners and at two different stages.

The focus of this study is on a magneto-thermomechanical coupled system. Consequently, the only common physical quantities taken into account are the magnetic force density, the Joule losses and the temperature. The force density is involved in the direct magnetomechanical coupling, whereas the Joule losses one is responsible for the magneto-thermal coupling. Since the material behavior varies with the temperature, the mechanical state also depends on the temperature leading to a third kind of coupling.

\subsubsection{Magneto-mechanical coupling}

The forces are obtained using the virtual power principle (VPP) in its Lagrangian form. This method has the advantage of computing Lorentz force and forces due to ferromagnetic parts in a unique process. Lorentz force suits well the homogeneous permeability case but does not take into account ferromagnetic forces [4] if materials of different permeabilities are present $[5,6]$. The VPP procedure is based on the local computation of the magnetic energy $(W)$ or magnetic co-energy $\left(W^{*}\right)$ defined as follows:

$$
W=\int_{\mathcal{D}} \mathbf{H} \cdot \mathrm{d} \mathbf{B} \text { and } W^{*}=\int_{\mathcal{D}} \mathbf{B} \cdot \mathrm{d} \mathbf{H} .
$$

Forces are deducted from one expression of the energy through a spatial differentiation:

$$
F_{s}=-\partial_{u} W \text { and } F_{s}=\partial_{u} W^{*} .
$$

Expression of forces using co-energy is adapted to the magnetic formulation, while the use of the energy is adapted to the electric formulation [7-11]. The choice of a formulation then imposes the expression of forces to be used. The " $s$ " subscript denotes that the computation has to be done for all elements surrounding the considered node. It therefore produces the nodal value of the force. To compute the force density $f$, the following formula must be used [12]:

$$
\mathbf{A f}=\mathbf{F} \text { and } A_{i j}=\int_{\mathcal{D}} w_{n_{i}} w_{n_{j}} .
$$

The $n$ subscript refers to nodal basis functions used for discretization. $[F]$ and $[f]$ matrices respectively contain degrees of freedom (DoF) of the nodal forces and of the force density.

\subsubsection{Magneto-thermal coupling}

Magnetic and thermal problems are linked by Joule losses which are involved in the assembly of the thermal system's right-hand side of the equation (19). For this coupling process we determine heat losses using the magnetic 
unknowns, before transferring them to the thermal problem. Joule losses are computed using the electric field ${ }^{7}$ or current density:

$$
s=\frac{|\mathbf{J}|^{2}}{\sigma}=\sigma|\mathbf{E}|^{2} .
$$

Using this equation, the thermal right-hand side can be computed and solved.

\subsubsection{Thermo-mechanical coupling}

The temperature distribution $(T)$ arises from the magnetic computation and is a prerequisite to the process of the mechanical problem. This kind of coupling is of different nature - compared with the magneto-mechanical oneas a behavior law modification is involved: the mechanical problem not only depends on the magnetic forces but also on the temperature through the material behavior law. As an immediate consequence the thermal problem must be solved before the mechanical one.

Once the thermal solution is computed, the mechanical deformation can be determined using the following behavior law:

$$
\sigma=\frac{E}{1+\nu}\left(\epsilon+\frac{\nu}{1-2 \nu} \operatorname{Tr}(\epsilon) G\right)-\frac{\alpha E}{1-2 \nu}\left(T-T_{r e f}\right) G,
$$

where $\alpha$ is the coefficient of thermal expansion and $T$ is the temperature. $T_{r e f}$ is a parameter accounting for an external temperature. The first part of the behavior law (Eq. (24)) is the classical Hooke law (see Eq. (9)). The additional expression in equation (24) takes into account the thermal phenomenon and can be seen as an equivalent load applied to the system. Consequently, thermomechanical coupling results in the addition of a term in the mechanical right-hand side of the equation (13).

\subsection{Disconnected meshes coupling}

\subsubsection{Introduction}

When the topology of the problem is not changed significantly, a successive resolution of each sub-problem is sufficient. On the other hand, if the modification ${ }^{8}$ of the magnetic state with the deformation cannot be neglected, the use of iterative coupling methods becomes essential.

As the problem domain might change, the availability of disconnected coupling methods is of great help. In consequences:

- Re-meshing process and code reuse are facilitated;

- The mesh type can be adapted to the physical problem improving the discretization efficiency;

\footnotetext{
7 The electric field was not considered in the magnetic model Nevertheless, eddy-current models were performed invoking this equation.

8 The use of iterative coupling is closely linked to the required accuracy.
}

- Each sub-problem converges better than its strongly coupled counterpart;

- The number of degrees of freedom is decreased (especially for linear cases).

Moreover, this weak coupling process makes the physical time steps easy to separate.

\subsubsection{Theoretical considerations}

The data transfer method is based on an orthogonal projection with the help of an adequate sub-space. The aim is to: establish the target function that best fits the source function according to the appropriate dot product. The distance between the source and the target functions is then minimized:

$$
u_{t}:=\forall u \in \mathcal{V} \in\left\|u_{s}-u_{t}\right\| \leq\left\|u_{s}-u\right\| .
$$

In the previous equation $u_{s}$ denotes the source function and $u_{t}$ is the target one. $\mathcal{V}$ is a so-called Banach functional space (e.g., $\mathcal{L}^{2}$ ) associated to the $\|$.$\| norm. The general u$ function can either be scalar or vectorial. Let $\mathcal{D}_{\text {com }}$ be a topological domain common to two physical models (e.g., $\overline{\mathcal{D}}_{\text {com }}=\overline{\mathcal{D}}_{\text {mag }} \cap \overline{\mathcal{D}}_{\text {mec }}$ ), then $\mathcal{V}$ is a functional space which refers to one of these particular spaces:

$$
\begin{aligned}
& \mathcal{L}_{\mathcal{D}_{\text {com }}}^{2}:\left\{u, \underset{\mathcal{D}_{\text {com }}}{\int} u^{2} \text { exists }\right\}, \\
& \mathcal{H}_{\mathcal{D}_{\text {com }}^{\text {grad }}}^{\text {gra }}:\left\{u \in \mathcal{L}_{\mathcal{D}_{\text {com }}}^{2}, \operatorname{grad} u \in \mathcal{L}_{\mathcal{D}_{\text {com }}}^{2}\right\}, \\
& \mathcal{H}_{\mathcal{D}_{\text {com }}}^{\text {curl }}:\left\{u \in \mathcal{L}_{\mathcal{D}_{\text {com }}}^{2}, \operatorname{curl} u \in \mathcal{L}_{\mathcal{D}_{\text {com }}}^{2}\right\}, \\
& \mathcal{H}_{\mathcal{D}_{\text {com }}^{\text {div }}}^{\text {div }}:\left\{u \in \mathcal{L}_{\mathcal{D}_{\text {com }}}^{2}, \operatorname{div} u \in \mathcal{L}_{\mathcal{D}_{\text {com }}}^{2}\right\} .
\end{aligned}
$$

For simplicity reasons the subscript relative to the domain will be omitted. In what follows, $\mathcal{D}_{\text {com }}$ is the common part to the two meshes independently of the considered physical models.

We add to these spaces inner products of two types. The first one, relative to the $\mathcal{L}^{2}$ space, is defined as:

$$
(u, v) \in \mathcal{L}^{2},\langle u, v\rangle_{\mathcal{L}^{2}}=\int_{\mathcal{D}_{c o m}} u \cdot v .
$$

The second one, generically called the $\mathcal{H}^{1}$ scalar product, ${ }^{9}$ is written as follows:

$$
\begin{aligned}
& (u, v) \in \mathcal{H}^{\text {grad }},\langle u, v\rangle_{\mathcal{H}^{\text {grad }}}=\underset{\mathcal{D}_{\text {com }}}{\int} u \cdot v+\operatorname{grad} u \cdot \operatorname{grad} v, \\
& (u, v) \in \mathcal{H}^{\text {curl }},\langle u, v\rangle_{\mathcal{H}^{\text {curl }}}=\int_{\mathcal{D}_{\text {com }}} u \cdot v+\operatorname{curl} u \cdot \operatorname{curl} v, \\
& (u, v) \in \mathcal{H}^{d i v},\langle u, v\rangle_{\mathcal{H}^{d i v}}=\int_{\mathcal{D}_{c o m}} u \cdot v+\operatorname{div} u \cdot \operatorname{div} v .
\end{aligned}
$$

${ }^{9}$ Although $\mathcal{H}^{\text {curl }}$ and $\mathcal{H}^{\text {div }}$ do not correspond to $\mathcal{H}^{1}$. Due to the use of Whitney elements, discretized functions actually belong to $\mathcal{H}^{1}$ and therefore to $\mathcal{H}^{\text {curl }}$ and $\mathcal{H}^{\text {div }}$. 
These dot products also define norms, in a general way we will define as:

$$
(u) \in \mathcal{X},\|u\|_{\mathcal{X}}=\sqrt{\langle u, u\rangle_{\mathcal{X}}},
$$

where $\mathcal{X}$ stands for one of the four presented sub-spaces. When $\mathcal{X}$ is the $\mathcal{L}^{2}$ space, we talk about the $\mathcal{L}^{2}$ norm. When $\mathcal{X}$ is either $\mathcal{H}^{\text {grad }}, \mathcal{H}^{\text {curl }}$, or $\mathcal{H}^{\text {div }}$, we refer to the $\mathcal{H}^{1}$ norm or the "energy norm".

To compute the target values of the function, we use the weak form of the following equation:

$$
u_{t}-u_{s}=0 .
$$

Using one of the so-defined dot products, the weak formulation is written:

$$
\left\langle u_{t}-u_{s}, \psi\right\rangle_{\mathcal{X}}=0, \forall \psi \in \mathcal{V}
$$

In general functions are discretized using specific basis functions (node elements, edge elements, face elements, etc.) and then belong to the finite counterpart of the cited sub-spaces. Two independent triangulations $\left(\mathcal{T}_{\text {com }}^{h_{s}}\right.$ the source triangulation and $\mathcal{T}_{\mathrm{com}}^{h_{t}}$ the target one) belong to the domain $\mathcal{D}_{\text {com }}$. We thus introduce the discrete functional framework using $\mathcal{V}_{s}$ and $\mathcal{V}_{t}$ which are respectively the finite spaces related to the source mesh $\mathcal{T}_{\text {com }}^{h_{s}}$ and the target mesh $\mathcal{T}_{\text {com }}^{h_{t}}$. Therefore $u_{s}$ and $u_{t}$ respectively belong to $\mathcal{V}_{s}$ and $\mathcal{V}_{t}$

The use of the traditional node, edge or face-based elements ensures that the discretized values belong to one of the presented sub-spaces. Functions are considered as distributions enabling the use of spatial derivatives. $u_{s}$ and $u_{t}$ are then expressed by:

$$
u_{s}=\sum_{i} u_{s_{i}} w_{s_{i}} \text { and } u_{t}=\sum_{i} u_{t_{i}} w_{t_{i}}
$$

where $w_{i}$ is the $i$ th basis function. Subscripts $s$ and $t$ respectively refer to the source or target mesh.

We now replace the space $\mathcal{V}$ by $\mathcal{V}_{t}$. We successively test equation (30) using the basis functions of the target mesh. This method is called the Ritz-Galerkin method [13] and leads to:

$$
\left\langle u_{t}-u_{s}, w_{t_{i}}\right\rangle_{\mathcal{X}}=0 \text { for all } w_{t_{i}} \in \mathcal{V}_{t}
$$

It must be solved for all $\psi=w_{t_{i}}$ selected from the targetmesh elements functions. $\mathcal{X}$ refers - in an abstract way and for compactness sake - to one of these sub-spaces: $\mathcal{L}^{2}, \mathcal{H}^{\text {grad }}, \mathcal{H}^{\text {curl }}$ or $\mathcal{H}^{\text {div }}$. As $\mathcal{V}_{t} \subset \mathcal{H}^{1}$ and $\mathcal{H}^{1} \subset \mathcal{L}^{2}$, we can use the inner product of $\mathcal{H}^{1}$ and the inner product of $\mathcal{L}^{2}$. For each kind of basis function the two inner products will be considered.

The Ritz-Galerkin method [13] is a very common process and is widely available in many FEM-related programs. The resulting consequences of the methods are advantageous:

- the target (projected) function is unique;

$-u_{s}-u_{t}$ belongs to the orthogonal sub-space of $\mathcal{V}_{t}$;

- $\left\|u_{s}-u_{t}\right\|$ is minimal within the chosen norm;
- the so-computed matrix is invertible, symmetric and positive-definite. This leaves a large choice for the solver.

Moreover, if $u_{s}$ is associated to a first mesh and $u_{t}$ is associated to a second mesh, $u_{s}-u_{t}$ has a finite dimension and directly depends on the number of volume intersections created by the overlapping meshes.

The overall conformity of the method is ensured by the discretization for both $u_{s}$ and $u_{t}$. However, test functions $\psi$ may not belong to one of the cited sub-spaces, leading to a non-conform Galerkin method. ${ }^{10}$

The Ritz-Galerkin method leads to a linear system of type:

$$
[M]\left[U_{t}\right]=[N]\left[U_{s}\right]
$$

where $\left[U_{t}\right]$ and $\left[U_{s}\right]$ are degrees of freedom of source and target functions. $[M]$ and $[N]$ are two mass-matrices assembled using a FEM-based loop over elements. As an example, in the $\mathcal{L}^{2}$ case, the respective elements are equal to:

$$
\begin{aligned}
M_{i, j} & =\int_{\left\{\mathcal{K}_{t}\right\}} w_{t_{i}} \cdot w_{t_{j}}, \\
N_{i, j} & =\int_{\left\{\mathcal{K}_{s}\right\} \leftrightarrow\left\{\mathcal{K}_{t}\right\}} w_{t_{i}} \cdot w_{s_{j}} .
\end{aligned}
$$

$\{\mathcal{K}\}$ represents all elements for which basis functions $w_{i} \cdot w_{j}$ are simultaneously nonzero. Obviously the sum is zero if such an element does not exist. $w_{s_{j}}$ is the $j$ th basis function of the source mesh $\left(u_{s}=\Sigma w_{s_{j}} u_{s_{j}}\right)$ defined by the triangulation $\mathcal{T}_{\text {com }}^{h_{s}}$. The symbolic representation $\left\{\mathcal{K}_{s}\right\} \leftrightarrow\left\{\mathcal{K}_{t}\right\}$ denotes that the sum has to be done over the sub-elements where $w_{t_{i}} \cdot w_{s_{j}}$ is not zero.

These sub-elements are generated by the intersection between the source and the target mesh. A fast intersection computation must be developed and is probably the main difficulty of this stage [14-16].

The projection method is actually applicable to any $u_{s}$ and is not restricted to the discretized source functions. This method can also be helpful to compute an approximation of any function in the desired discrete sub-space. The following equation is used: $[M]\left[U_{t}\right]=\left[U_{a}\right]$, where $U_{a_{i}}$ $=\int w_{i} \cdot u_{s}$. A single mesh is then considered, and $\left[U_{t}\right]$ is now the array of degrees of freedoms (DoFs) of the discretized counterpart of the function $u_{s}$.

When $u$ is a vector and is discretized using node elements (e.g., force density projection), equation (34) is solved a number of times equal to the number of components. The array $\left[U_{s}\right]$ successively represents the Dofs along each direction.

\subsection{Strategies for accurate coupling methods}

We present in this part the general process involved in the magneto-mechanical coupling. The aim is to solve the

10 This case is not considered in the present method. 
(a)

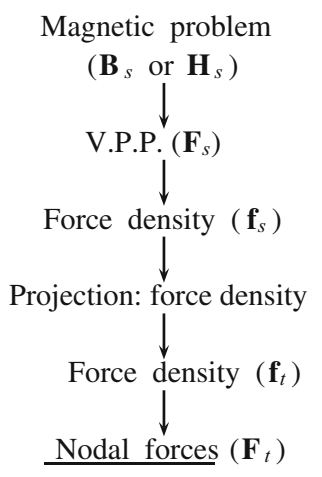

(b)

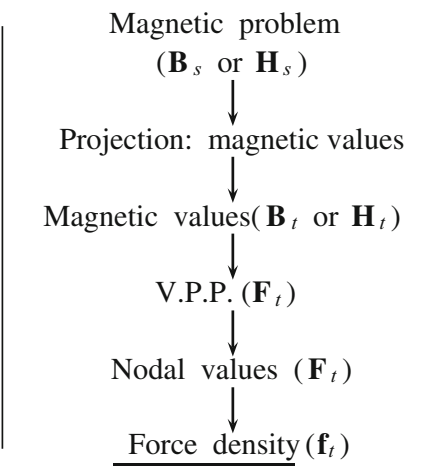

Fig. 3. Main coupling strategies: force density projection vs. magnetic quantities projection. Subscripts $s$ or $t$ respectively refer to the source mesh and the target mesh.

magnetic and mechanical models with the help of two different computer programs. This operation requires the application of the projection method presented in Paragraph 1.5.2. Unfortunately, the choice of a coupling variable is not unique.

Because of the high number of possible choices for the magneto-thermo-mechanical coupling, we have deliberately restricted this study to the magneto-mechanical case. Similar considerations apply to the magneto-thermomechanical model, for which the most straightforward way to couple the different sub-problems is the use of nodal projections involving the Joule losses and temperature.

\subsubsection{Predictive process}

Projection chains. Forces are source data for the mechanical problem. Two main procedures can be applied as summed up in Figure 3:

- projection of force density from magnetism to mechanics using precomputed forces;

- determination of forces using projected magnetic quantities.

A first approach consists in the application of the VPP followed by the projection of the force density (see Fig. 3b).

Another approach of the magneto-mechanical coupling relies on the projection of magnetic values over the second mesh. This method was tested as presented in reference [17]. The VPP is then applied within the mechanical mesh. In addition, magnetic projected quantities can be, depending on the formulation, one among the pair $\Omega$ and $\mathbf{H}$ or $\mathbf{A}$ and $\mathbf{B}$. Node elements or edge elements are used respectively for the magnetic formulation, and edge elements or facet elements are used for the electric one.

Because the mechanical program accepts both force density or nodal values, underlined processes in Figure 3 are optional stages.

\subsubsection{Corrective process and re-meshing}

In mechanics most functions are interpolated with the help of node elements. The displacement field is brought back

from the mechanical domain to the magnetic one using projections and nodal basis functions.

This allows an update of the geometry of each mesh. While the resort to a single mesh imposes the deformation of the elements according to the displacement, the projection method facilitates the re-meshing process as no assumption is made on the meshes. This operation has to be done with care to avoid unwanted shape modifications.

\subsection{Conclusion about the coupling method}

Spatial restriction. The projection process takes part in the mechanical source computation: it is composed of a force density computation stage and a projection stage. However, these processes are only useful in the "hard materials". The spatial restriction can improve the process as for example when all elements which are not essential to the computation are withdrawn: the memory cost of the projection method and the numerical models are reduced. We also have noticed that the restriction significantly improves conditioning of the systems of the type of equation (34).

Physical application of the projections. An innovative aspect of this work lies in the use of the projection methods as a foundation for multi-physics system modeling. Computer tools dedicated to the computation of one physics are widely available. However, computation code dedicated to a specific set of physics is uncommon. The projection method enables the intercommunication between computational tools. The ways of combining them, and thus the possibility to model coupled problems, are therefore endless.

\section{Numerical application of the projection methods}

\subsection{Projection tests}

The aim of numerical applications is to perform accuracy tests of the projection methods. To achieve these tests we have used successively node elements, edge elements and face elements. Two meshes has been considered in order to determine the most efficient projection process. We have defined an analytic function which allows the evaluation of the discretization and projection errors. This function is well defined for each mesh, then producing an unbiased source.

Procedure. We started with an analytical function $u$ as source data. In order to separate the discretization error from the projection error, the test is divided into the two following parts. 


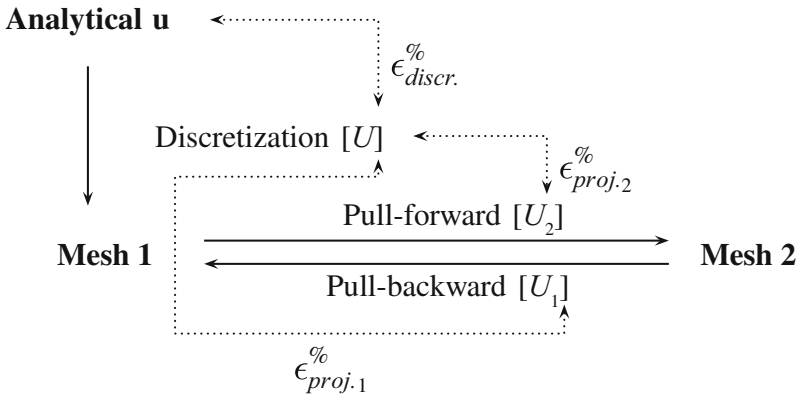

Fig. 4. Details of the discretization stage, the pull-forward projection, and the pull-backward projection. $[U]$ is compared with $u$ while $\left[U_{1}\right]$ and $\left[U_{2}\right]$ are compared with $[U]$.

- The first stage consists in computing a source vector $[U]$ stemming from the discretization of $u$ (a projection method was used). $[U]$ is computed by solving the equation:

$$
[M][U]=[S],
$$

where matrices are, in the $\mathcal{L}^{2}$ case:

$$
\begin{aligned}
M_{i, j} & =\int_{\mathcal{D}_{\text {com }}} w_{i} \cdot w_{j}, \\
S_{i} & =\int_{\mathcal{D}_{c o m}} w_{i} \cdot u .
\end{aligned}
$$

- In a second stage, the error committed when transferring a function from one mesh to another is computed. Starting from the reference array $[U]$ we have transferred the discretized function over a second mesh. The first mesh (the finest one, the second mesh being coarser) remains the same as used for the discretization part. Meshes are supposed to be disconnected (no relationship between them). This projection is called the pull-forward projection.

- The final stage consists in bringing back the solution over the first mesh. This projection is called the pullbackward projection. The different projection and error measurement stages are summarized in Figure 4.

Error measurement. As detailed in Paragraph 1.5.2, functions discretized using node elements, edge elements and face elements belong respectively to $\mathcal{H}^{\text {grad }}, \mathcal{H}^{\text {curl }}$ and $\mathcal{H}^{\text {div }}$. This means that they also belong to the $\mathcal{L}^{2}$ functional space. For each kind of element, two inner products can be considered: one relative to $\mathcal{H}^{1}$ and one relative to $\mathcal{L}^{2}$. The norm used to evaluate the error is chosen in accordance with the dot product.

Despite the fact that the Galerkin method ensures that the projected function is the most accurate one - relative to the inner product - errors can be measured using both the norms relative to $\mathcal{L}^{2}$ and to $\mathcal{H}^{1}$. This is helpful to assess the influence of the inner product on the projection method. (a)

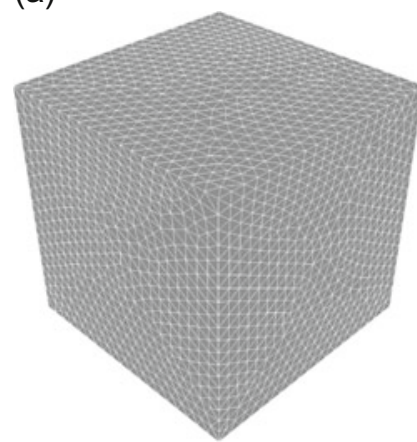

(b)

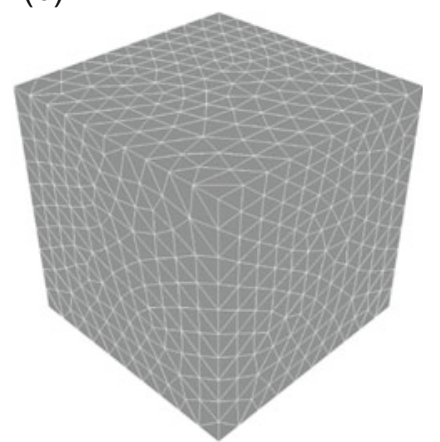

Fig. 5. Meshes used for projection tests. Functions are initially discretized through the fine mesh (a).

Errors, presented in percent of the norm, are computed as follows:

$$
\epsilon_{\mathcal{X}}^{\%}=\frac{\left\|u_{p}-u_{r}\right\|_{\mathcal{X}}}{\left\|u_{r}\right\|_{\mathcal{X}}},
$$

where $\mathcal{X}$ can be $\mathcal{L}^{2}$ or, depending on the element kind, $\mathcal{H}^{\text {grad }}, \mathcal{H}^{\text {curl }}$ and $\mathcal{H}^{\text {div }}$. $u_{r}$ represents the reference solution while $u_{p}$ is the one coming from the projection method.

Details. Once transferred to the second mesh, the reference and projected functions no longer belong to the same mesh. In order to correctly compute the projection error, actual values of the reference function are computed through the second mesh. This require an extra treatment of the intersection between the meshes. The projection error actually depends on the solely mesh topology.

The projection method will be applied to the inner product of $\mathcal{L}^{2}$ and the one of $\mathcal{H}^{1}$. In order to analyze the influence of the dot product on the error, $\epsilon_{\mathcal{X}}^{\%}$ will be computed using both the $\mathcal{L}^{2}$ and $\mathcal{H}^{\text {grad }}$ norms.

The assembly of the array $[U]$ always stems from the $\mathcal{L}^{2}$ projection of the analytical function $u$. In a real case $[U]$ is an intermediate array generated by a finite elements (FEM) program and may not present any particular characteristics. We have only used the analytical value of $u$ in order to assemble the source array and to assess the discretization error.

Meshes. Meshes are based on a cube of one unit edge length (see Fig. 5). As the distance unit does not matter, every value is given per unit. The first mesh (the fine one) is composed of 31000 linear tetrahedra $(h=0.06)$ while the second one (the coarser one) is composed of 6000 elements $(h=0.1)$. $h$ is called the characteristic mesh size as it characterizes the fineness of the mesh. This value typically represents the maximal diameter of the elements.

In the case of nodal values projections, the target mesh can also support quadratic elements (ten-nodetetrahedra). At the time of writing the software used for the projection does not support quadratic edge or face elements. 
The test consists in computing the global error compared to the reference vector $[U]$ at each projection stage. As the $\mathcal{L}^{2}$ and $\mathcal{H}^{1}$ projection methods can be used, at each stage the values of $\epsilon_{\mathcal{L}^{2}}^{\%}$ and $\epsilon_{\mathcal{H}^{1}}^{\%}$ have been computed. It represents the global error computed for the two methods using the respective norms. In order to evaluate the evolution of the errors for multiple computations, a test-case of 50 iterations was performed. An iteration merely symbolizes a pair of pull-forward and pull-backward projections.

\subsubsection{Node elements}

This paragraph deals with functions discretized with the help of node elements. As presented in Paragraph 1.5.2 these functions belong to $\mathcal{H}^{\text {grad }}$. Mathematically the appropriate dot product is:

$$
\langle u, v\rangle_{\mathcal{H}^{\text {grad }}}=\int_{\mathcal{D}_{\text {com }}} u \cdot v+\operatorname{grad} u \cdot \operatorname{grad} v .
$$

As $\mathcal{H}^{\text {grad }}$ is a part of the $\mathcal{L}^{2}$ functional space, we also deal with the corresponding dot product. It merely represents the first term in equation (39): $\int u \cdot v$.

The discretization method and the projection method are applied to the following analytical function:

$$
\begin{aligned}
u & =r^{3}+r+1, \\
\operatorname{grad} u & =\left(3 r^{2}+1\right) \mathbf{u}_{r},
\end{aligned}
$$

where $\mathbf{u}_{r}$ is the radial unit vector of the local spherical system. This function also presents $\mathcal{L}^{2}$-like properties.

This work is a part of multi-physics modeling methods. As the process will involve nodal quadratic basis functions, we will exceptionally deal with this case. Therefore results are separated into two parts: the linear case and the quadratic case.

Linear basis functions. Each mesh is composed of fournode-tetrahedra supporting linear basis functions. Table 1 presents discretization errors. Results for the first pullforward and pull-backward projection are presented in Table 2. Errors are thus computed using the discretized reference value of the function $[U]$ issued from an $\mathcal{L}^{2}$ projection.

Figures 6 and 7 (solid line) present a plot of the error over 50 pairs of pull-forward and pull-backward projections.

Table 1. Value of $\epsilon_{\text {discr. }}^{\%}$ the discretization error for linear node elements.

\begin{tabular}{ccc}
\hline & \multicolumn{2}{c}{$\epsilon_{\text {discr. }}^{\%}$ Norm type } \\
\cline { 2 - 3 } Projection & $\mathcal{L}^{2}$ & $\mathcal{H}^{\text {grad }}$ \\
\hline $\mathcal{L}^{2}$ & 0.0133 & 1.9572 \\
$\mathcal{H}^{\text {grad }}$ & 0.0212 & 1.9396 \\
\hline
\end{tabular}

Table 2. Values of $\epsilon_{p r o j .1}^{\%}$ and $\epsilon_{p r o j .2}^{\%}$ for linear node elements.

\begin{tabular}{|c|c|c|c|c|}
\hline \multirow[b]{2}{*}{ Projection } & \multicolumn{2}{|c|}{$\epsilon_{\text {proj.2 }}^{\%}$ Norm type } & \multicolumn{2}{|c|}{$\epsilon_{\text {proj.1 }}^{\%}$ Norm type } \\
\hline & $\mathcal{L}^{2}$ & $\mathcal{H}^{\text {grad }}$ & $\mathcal{L}^{2}$ & $\mathcal{H}^{\text {grad }}$ \\
\hline $\mathcal{L}^{2}$ & 0.1231 & 5.1577 & 0.0881 & 2.7512 \\
\hline $\mathcal{H}^{\text {grad }}$ & 0.1284 & 5.1354 & 0.0803 & 1.7950 \\
\hline
\end{tabular}
$\epsilon_{p r o j}^{\%}$ is the error between the projected value of the function and the reference array.

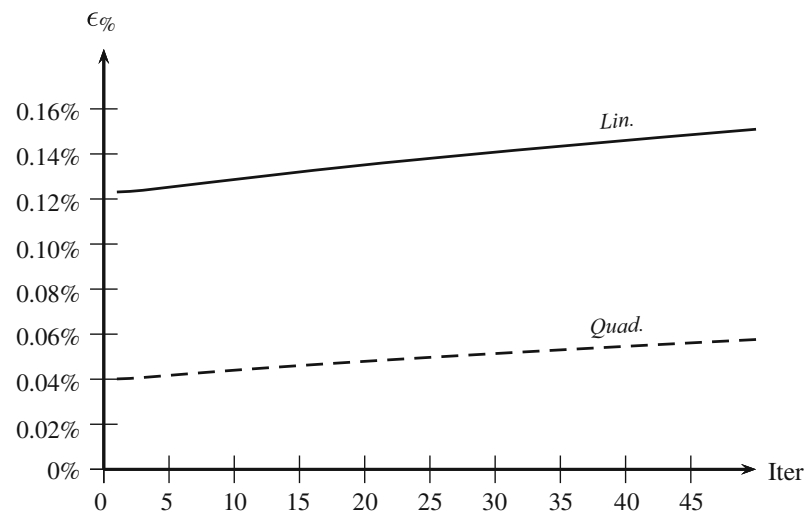

Fig. 6. Evolution of the projection error $\epsilon_{\text {proj. } 2}^{\%}$ for either linear or quadratic target basis functions. Results are given for the $\mathcal{L}^{2}$ inner product.

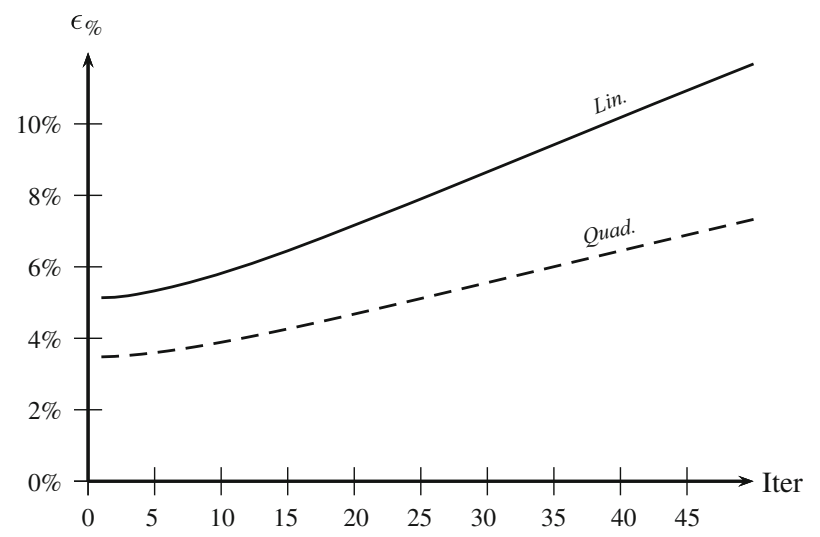

Fig. 7. Evolution of the projection error $\epsilon_{\text {proj.2 }}^{\%}$ for either linear or quadratic target basis functions. Results are given for the $\mathcal{H}_{\text {grad }}$ inner product.

Quadratic basis functions. The same tests were performed with a second mesh supporting quadratic basis functions. The first mesh still supports linear basis functions. The geometry of the second mesh has not changed; linear elements have just been replaced with quadratic ones. Table 3 details errors for the first iteration. Figures 6 and 7 detail the evolution of the error (dashed line) for 50 iterations (pull-forward/pull-backward projections pair).

Results show that the solution is better preserved with the use of quadratic elements as it was expected considering the higher number of degrees of freedom used in this case. 
Table 3. Values of $\epsilon_{p r o j .1}^{\%}$ and $\epsilon_{p r o j .2}^{\%}$, the projection error, in the case where the second mesh supports quadratic basis functions.

\begin{tabular}{ccccc}
\hline & \multicolumn{2}{c}{$\epsilon_{\text {proj.2 }}^{\%}$} & Norm type & \multicolumn{2}{c}{$\epsilon_{\text {proj.1 }}^{\%}$} & Norm type \\
\cline { 2 - 5 } Projection & $\mathcal{L}^{2}$ & $\mathcal{H}^{\text {grad }}$ & $\mathcal{L}^{2}$ & $\mathcal{H}^{\text {grad }}$ \\
\hline $\mathcal{L}^{2}$ & 0.0401 & 3.4952 & 0.0066 & 0.3181 \\
$\mathcal{H}^{\text {grad }}$ & 0.0425 & 3.4799 & 0.0279 & 0.3115 \\
\hline
\end{tabular}

Table 4. Discretization $\left(\epsilon_{d i s c r}^{\%}\right)$ error for edge elements.

\begin{tabular}{ccc}
\hline & \multicolumn{2}{c}{$\epsilon_{\text {discr. }}$ Norm type } \\
\cline { 2 - 3 } Projection & $\mathcal{L}^{2}$ & $\mathcal{H}^{\text {rot }}$ \\
\hline $\mathcal{L}^{2}$ & 1.6929 & 24.040 \\
$\mathcal{H}^{\text {rot }}$ & 7.2256 & 4.4703 \\
\hline
\end{tabular}

The Galerkin method produces the optimal solution according to the chosen inner product. Therefore it is not surprising that the $\mathcal{H}^{\text {grad }}$ method slightly reduces the $\mathcal{H}^{\text {grad }}$ norm of the error while slightly raising the $\mathcal{L}^{2}$ norm. An advantage of the $\mathcal{H}^{\text {grad }}$ method lies in the control over spatial derivatives.

These first two results show that the transfer of nodal values is better ensured with the use of quadratic basis functions. However, the inner product, in the case of node elements, has little influence on the projection error.

\subsubsection{Edge elements}

The next accuracy test provides error measurement for functions discretized using edge elements. Similarly to the first test (Sect. 2.1.1), the initial (reference) array represents the circulations of the analytical function along the edges. The analytical value of the field uses the spherical coordinates system and is directed along the azimuthal unit vector. In order to avoid singularities of the curl operator, an extra term directed along the radial unit vector has been added. We provide the expressions of the function and its curl:

$$
\begin{aligned}
\mathbf{u} & =\mathbf{u}_{r}+\left(r^{3}+r\right) \sin (\theta) \mathbf{u}_{\phi}, \\
\operatorname{curl} \mathbf{u} & =2\left(r^{2}+1\right) \cos (\theta) \mathbf{u}_{r}+\left(4 r^{2}+2 r\right) \sin (\theta) \mathbf{u}_{\theta} .
\end{aligned}
$$

Expressions are given using the local spherical coordinates system, where $\theta$ is the azimuthal angle.

As we consider linear basis functions, only two tests can be led. The first one uses the $\mathcal{L}^{2}$ dot product whereas the second one uses the $\mathcal{H}^{\text {curl }}$ one.

As for the node elements, Tables 4 and 5 present errors for the discretization and the projection stages respectively. Figure 8 is an overview of the evolution of the error for multiple projections.

\subsubsection{Face elements}

Finally face-based discretized functions are considered in this paragraph. This last test uses the $\mathcal{H}^{\text {div }}$ norm. The ref-
Table 5. Values of $\epsilon_{p r o j .1}^{\%}$ and $\epsilon_{p r o j .2}^{\%}$ : the projection error for edge elements.

\begin{tabular}{ccccc}
\hline & \multicolumn{2}{c}{$\epsilon_{\text {proj.2 }}^{\%}$} & Norm type & \multicolumn{2}{c}{$\epsilon_{\text {proj.1 }}^{\%}$} & Norm type \\
\cline { 2 - 5 } Projection & $\mathcal{L}^{2}$ & $\mathcal{H}^{\text {rot }}$ & $\mathcal{L}^{2}$ & $\mathcal{H}^{\text {rot }}$ \\
\hline $\mathcal{L}^{2}$ & 4.0281 & 17.3093 & 1.8318 & 19.949 \\
$\mathcal{H}^{\text {rot }}$ & 4.0707 & 12.725 & 1.6408 & 11.878 \\
\hline
\end{tabular}

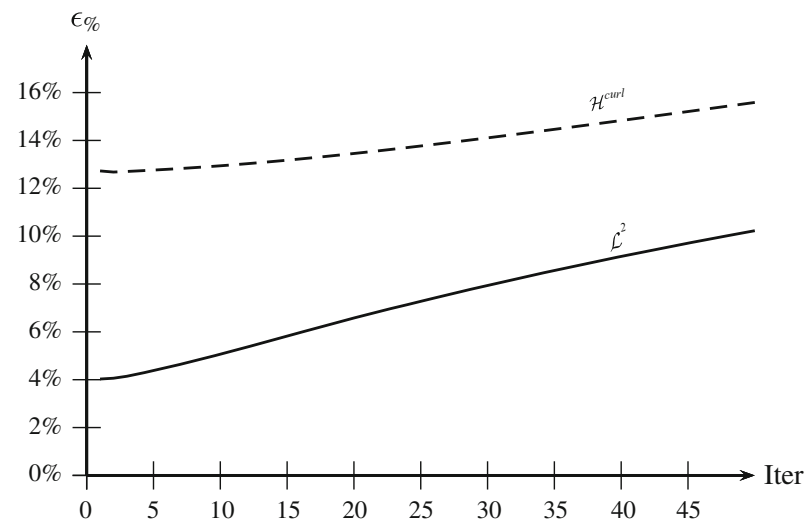

Fig. 8. Evolution of the projection error $\epsilon_{\text {proj.2 }}^{\%}$ (throughout the second mesh) in the case of edge elements. Results are given for both $\mathcal{L}^{2}$ and $\mathcal{H}_{\text {curl }}$ dot products. The second mesh supports linear elements only.

Table 6. Discretization error $\left(\epsilon_{\text {discr. }}^{\%}\right)$ for face elements.

\begin{tabular}{ccc}
\hline & \multicolumn{2}{c}{$\epsilon_{\text {discr. }}^{\%}$ Norm type } \\
\cline { 2 - 3 } Projection & $\mathcal{L}^{2}$ & $\mathcal{H}^{\text {div }}$ \\
\hline $\mathcal{L}^{2}$ & 1.8824 & 8.9880 \\
$\mathcal{H}^{\text {div }}$ & 1.9138 & 1.6431 \\
\hline
\end{tabular}

Table 7. Values of $\epsilon_{p r o j .1}^{\%}$ and $\epsilon_{p r o j .2}^{\%}$ : the projection errors for face elements.

\begin{tabular}{ccccc}
\hline & \multicolumn{2}{c}{$\epsilon_{\text {proj.2 }}^{\%}$} & Norm type & \multicolumn{2}{c}{$\epsilon_{\text {proj.1 }}^{\%}$} & Norm type \\
\cline { 2 - 5 } Projection & $\mathcal{L}^{2}$ & $\mathcal{H}^{\text {grad }}$ & $\mathcal{L}^{2}$ & $\mathcal{H}^{\text {div }}$ \\
\hline $\mathcal{L}^{2}$ & 4.4943 & 12.659 & 2.2532 & 11.917 \\
$\mathcal{H}^{\text {div }}$ & 4.5128 & 9.6621 & 2.2030 & 8.6635 \\
\hline
\end{tabular}

erence array stems from the discretization of the reference function. Its computation reflects the values of the flux across faces of the function $u$. Like the previous test, we have used the $\mathcal{L}^{2}$ and the $\mathcal{H}^{1}$ norms applied to the $\mathcal{L}^{2}$ and $\mathcal{H}^{\text {div }}$ sub-spaces. We provide expressions of the reference function $u$ and its divergence:

$$
\begin{aligned}
\mathbf{u} & =\left(r^{3}+r^{2}\right) \mathbf{u}_{r}+\mathbf{u}_{\phi} \\
\operatorname{div} \mathbf{u} & =\left(5 r^{2}+4 r\right)
\end{aligned}
$$

Discretization errors can be found in Table 6 while Table 7 details values of the projection error for the first iteration. The evolution of the error is presented in Figure 9. 


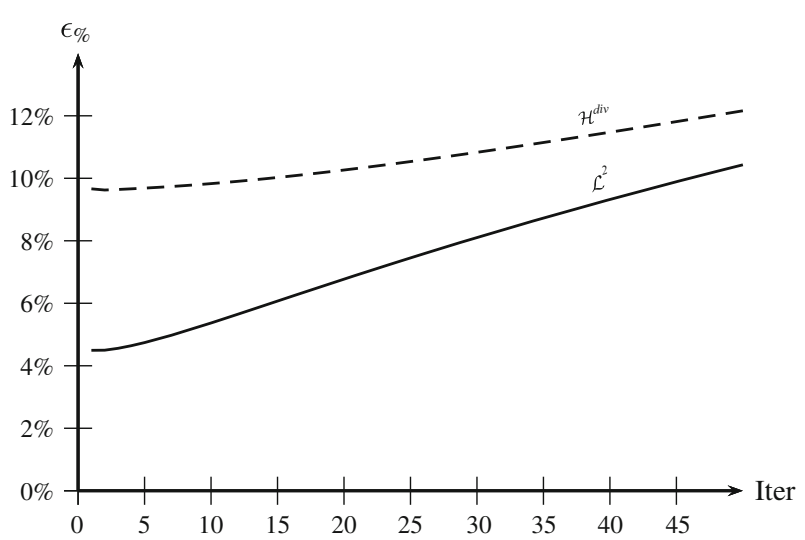

Fig. 9. Evolution of the projection error $\epsilon_{\text {proj.2 }}^{\%}$ in the case of face elements. Results are given for both $\mathcal{L}^{2}$ and $\mathcal{H}_{\text {div }}$ dot products.

Table 8. Values of $\epsilon_{p r o j .1}^{\%}$ and $\epsilon_{p r o j .2}^{\%}$ in the case of affiliated meshes.

\begin{tabular}{cccc}
\hline$\epsilon_{\text {proj.1 }}^{\%}$ & $\epsilon_{\text {proj.2 }}^{\%}$ & $\epsilon_{\text {proj.12 }}^{\%}$ & $\epsilon_{\text {proj.2. }}^{\%}$ \\
\hline 1.2345 & 1.2345 & 1.2345 & 1.2345 \\
\hline
\end{tabular}

\subsubsection{Discussion}

This method has resorted to three-dimensional overlapping meshes. To the best of knowledge, a priori upper bounds of the error has been studied for node elements only [18]. In that case, the error is bounded by a sum of values depending on the fineness of both the meshes. Despite its great similarities, theoretical results available for the mortar method do not apply here. This mortar method has extensively been studied but deals with meshes for which surfaces or lines overlaps [19-22].

As the two meshes define the source and the target discrete functional spaces, thanks to the Galerkin method, the error is entirely driven by the geometry of the triangulations. At the $n$th iteration, the square of the error $\left(\epsilon^{2}\right)$ is given by:

$$
F^{\top}\left[\left(M_{s}^{-1} N^{t} M^{-1} N\right)^{n \top}-I\right]\left[\left(M_{s}^{-1} N^{t} M^{-1} N\right)^{n}-I\right] F,
$$

where $M_{s}$ is the counterpart of the mass matrix $M_{t}$ but for source basis functions $\left(M_{s_{i j}}=\int w_{s_{i}} \cdot w_{s_{j}}\right)$. It obviously only depends on the triangulations and the array $[F]$. More thorough tests show that, for a given maximal diameter of the elements $(h)$, results on the error vary widely. This result is more outstanding when the fine mesh is a subdivision of the first one. Table 8 presents the first four iterations to which meshes are affiliated.

As the second functional space is fully included in the first one, all the error is obtained when passing from the fine mesh to the coarse one. Ordinary disconnected meshes will produce a constantly growing error.

We have also noticed that the way the reference vector $[U]$ is built is important. In the case where $[U]$ is assembled using a $\mathcal{H}^{1}$ projection, the relative error $\epsilon_{\mathcal{L}^{2}}^{\%}$ is not significantly modified whereas the value of $\epsilon_{\mathcal{H}^{1}}^{\%}$ is decreased.
Table 9. Value of $\epsilon_{\text {proj.2 }}^{\%}$ : the relative error for the first pullforward projection. Only the second mesh is modified.

\begin{tabular}{ccccccc}
\hline & \multicolumn{2}{c}{ Nodes } & \multicolumn{2}{c}{ Edges } & \multicolumn{2}{c}{ Faces } \\
\cline { 2 - 7 }$h_{2}$ & $\mathcal{L}^{2}$ & $\mathcal{H}^{\text {grad }}$ & $\mathcal{L}^{2}$ & $\mathcal{H}^{\text {curl }}$ & $\mathcal{L}^{2}$ & $\mathcal{H}^{\text {div }}$ \\
\hline 0.20 & 1.000 & 1.000 & 1.000 & 1.000 & 1.000 & 1.000 \\
0.17 & 0.520 & 0.742 & 0.724 & 0.955 & 0.746 & 0.922 \\
0.15 & 0.357 & 0.654 & 0.671 & 0.953 & 0.684 & 0.901 \\
0.13 & 0.344 & 0.641 & 0.645 & 0.937 & 0.665 & 0.882 \\
0.10 & 0.259 & 0.573 & 0.578 & 0.925 & 0.594 & 0.855 \\
0.07 & 0.101 & 0.422 & 0.438 & 0.921 & 0.459 & 0.834 \\
0.05 & 0.086 & 0.392 & 0.403 & 0.898 & 0.417 & 0.813 \\
\hline
\end{tabular}

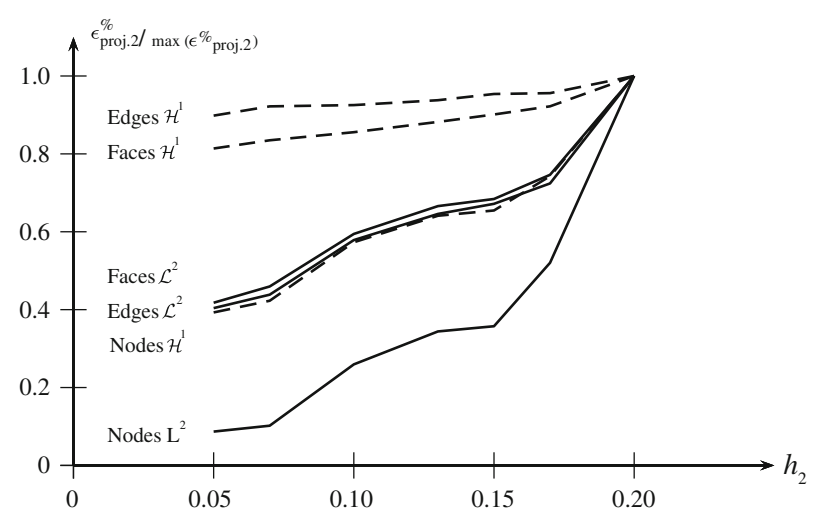

Fig. 10. Evolution of the projection error $\left(\epsilon_{\text {proj.2 }}^{\%}\right)$ with the characteristic mesh size $h_{2}$ for node, edge and face elements. $h_{1}$ is kept constant to $h_{1}=0.06$. Solid line: $\mathcal{L}^{2}$ case. Dashed: $\mathcal{H}^{1}$ case. For each case, values of the errors are normalized by the maximal one (maximal value of each column).

\section{Evolution of the error with the characteristic mesh size.}

In order to provide a simple asymptotic rate of the relative error, computations have been performed for several characteristic sizes. We denote by $h_{1}$ and $h_{2}$ the mesh characteristic sizes for the source and the target meshes respectively. Table 9 and Figure 10 presents results for an evolution of $h_{2}$ while the source mesh is kept constant to $h_{1}=0.06$. In that case, the projection error is essentially driven by the characteristic size of the second mesh.

Table 10 provides the evolution of the error for a joint evolution of meshes. The ratio of the characteristic sizes is kept constant to $h_{1} / h_{2}=5$. In the case of node elements, results are in agreement with the bound of the error given in reference [18].

Conclusion on field projection. The method to be chosen depends on the type of elements discretizing the function. In a very mathematical way, the preferred method has to be the one that uses the appropriate dot product regarding the sub-space. The analysis of the plot of the field $u$ can be misleading as the field seems less modified when $\mathcal{L}^{2}$ norm is used in comparison with the $\mathcal{H}^{1}$ norm. However, the projected solution better fits the $\mathcal{H}^{\text {grad }}, \mathcal{H}^{\text {curl }}$ or $\mathcal{H}^{\text {div }}$ norm even if the distribution of the field is visually 
Table 10. Value of $\epsilon_{p r o j .2}^{\%}$ : the relative error for the first pullforward projection. Mesh size ratio is kept constant to 5 .

\begin{tabular}{ccccccc}
\hline & \multicolumn{2}{c}{ Nodes } & \multicolumn{2}{c}{ Edges } & \multicolumn{2}{c}{ Faces } \\
\cline { 2 - 7 }$h_{2}$ & $\mathcal{L}^{2}$ & $\mathcal{H}^{\text {grad }}$ & $\mathcal{L}^{2}$ & $\mathcal{H}^{\text {curl }}$ & $\mathcal{L}^{2}$ & $\mathcal{H}^{\text {div }}$ \\
\hline 0.20 & 0.474 & 9.709 & 7.419 & 12.00 & 8.016 & 8.483 \\
0.17 & 0.235 & 6.796 & 5.169 & 9.113 & 5.741 & 6.054 \\
0.13 & 0.156 & 5.872 & 4.573 & 7.669 & 5.061 & 5.255 \\
0.10 & 0.119 & 5.060 & 3.997 & 6.601 & 4.408 & 4.504 \\
0.07 & 0.042 & 3.068 & 2.516 & 4.036 & 2.864 & 2.737 \\
\hline
\end{tabular}

modified. The use of $\mathcal{H}^{1}$ inner product significantly improves values of the spatial derivatives as it includes their contributions in the projection stage.

The legitimacy of the use of these two dot-products is questionable. The projection of potentials with the $\mathcal{L}^{2}$ norm is meaningful but does not take into account the associated field. Furthermore, the use of the $\mathcal{H}^{1}$ norm combines two values having two distinct physical meanings. The first term $\int \mathbf{f} \cdot \mathbf{g}$ represents the $\mathcal{L}^{2}$ projection of the potentials. The second term $\int d(\mathbf{f}) \cdot d(\mathbf{g})$ where $d$ can be grad, div or curl is the $\mathcal{L}^{2}$ projection of a physically significant field. The next section discusses this problem.

\subsection{Relative errors on physical fields}

\subsubsection{Introduction}

We will now determine which of the $\mathcal{L}^{2}$ and $\mathcal{H}^{1}$ projection methods, applied to the field or values the potentials, better preserves the solution. To answer this a second set of tests was performed. For each class of elements previous projection methods were reused. We added a third method which relies on the second term of generic $\mathcal{H}^{1}$ norm: $\int \operatorname{grad} u \cdot \operatorname{grad} v, \int \operatorname{curl} u \cdot \operatorname{curl} v$ for respectively nodes or edges.

This third method represents the $\mathcal{L}^{2}$ minimization between the physical meaningful electromagnetic fields, deduced from their respective potential. The accuracy criterion involves the final electromagnetic field computed throughout the second mesh.

We then introduce another inner product and the associated norm defined as:

$$
\begin{gathered}
(u, v) \in \mathcal{H}^{\text {grad }},\langle u, v\rangle_{\text {grad.grad }}=\iint_{\mathcal{D}_{c o m}} \operatorname{grad} u \cdot \operatorname{grad} v, \\
(u) \in \mathcal{H}^{\text {grad }},\|u\|_{\text {grad.grad }}=\sqrt{\langle u, u\rangle_{\text {grad.grad }}}
\end{gathered}
$$

The same thing is applied to the curl operator leading to the $\langle u, v\rangle_{\text {curl.curl }}$ product and the $\|u\|_{\text {curl.curl }}$ norm.

If a function $u$ represents a magnetic potential, then the projection of the magnetic field using the minimization between magnetic potential is equivalent to solving:

$$
\left\|u_{t}-u_{s}\right\|_{\mathcal{L}^{2}}=0
$$

whereas the distance minimization between magnetic fields is:

$$
\left\|u_{t}-u_{s}\right\|_{\text {grad } \cdot \operatorname{grad}}=0
$$

For each kind of element we will compare the error on the magnetic field values. We have successively computed the relative error defined by:

$$
\epsilon_{\mathcal{X}}^{\%}=\frac{\left\|u_{p}-u_{r}\right\|_{\text {grad } \cdot g r a d}}{\left\|u_{r}\right\|_{\text {grad.grad }}},
$$

when the electromagnetic potential is projected using both the $\mathcal{L}^{2}$ and the $\mathcal{H}^{1}$ inner products. $u_{p}$ and $u_{r}$ respectively refer to the projected and reference solutions.

The same tests were performed using edge elements. In this case the $\|\cdot\|_{\text {curl.curl }}$ was used.

\subsubsection{Projection of scalar potential}

Figure 11 presents computation results for node elements, the first iteration is detailed in Table 11. This method can be applied to the magnetic scalar potential $\Omega$ (node-based discretization) projected using both $\mathcal{L}^{2}$ and the $\mathcal{H}^{\text {grad }}$ functional spaces. Then the error over the magnetic field $\mathbf{H}$ is computed. Except for the three first iterations, the best results are obtained using the $\mathcal{L}^{2}$ norm for potentials.

\subsubsection{Projection of vector potential}

In a very similar way, computation results for edge elements are available in Figure 12 while Table 12 presents details on the first iteration. This method can be applied to the magnetic vector potentials $(\mathbf{A})$ while the error on associated magnetic induction $(\mathbf{B})$ is computed. The benefit of the $\mathcal{H}^{\text {curl }}$ dot product appears clearly: the spatial derivatives of the potential are greatly improved.

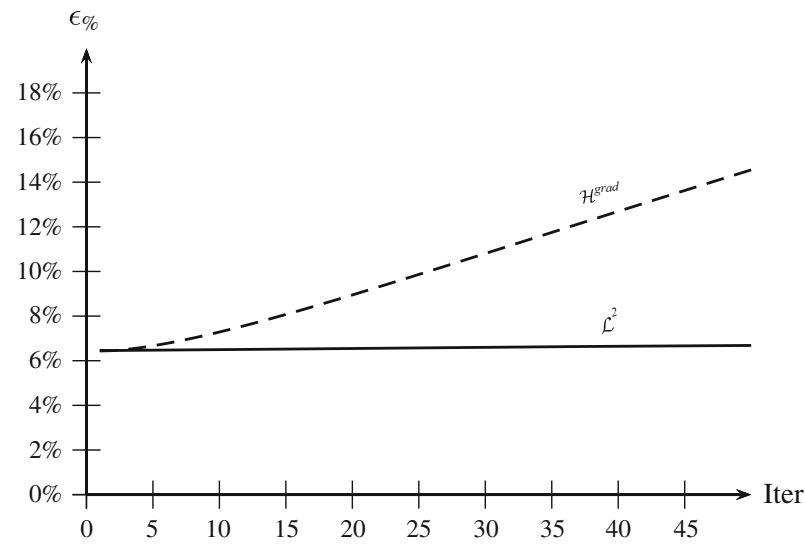

Fig. 11. Relative error in both $\mathcal{L}^{2}$ and $\mathcal{H}^{1}$ projection of node elements. Errors are evaluated using the distance between magnetic field $\left(\|\cdot\|_{\text {grad.grad }}\right)$.

Table 11. Values of $\epsilon_{p r o j .2}^{\%}$ : the error between the projected values over the second mesh for the first iteration.

\begin{tabular}{cc}
\hline Projection & Error $\left(\epsilon_{\text {grad.grad }}^{\%}\right)$ \\
\hline $\mathcal{L}^{2}$ & 6.4650 \\
$\mathcal{H}^{\text {grad }}$ & 6.4371 \\
\hline
\end{tabular}


A.A. Journeaux et al.: Multi-physics problems computation using numerically adapted meshes

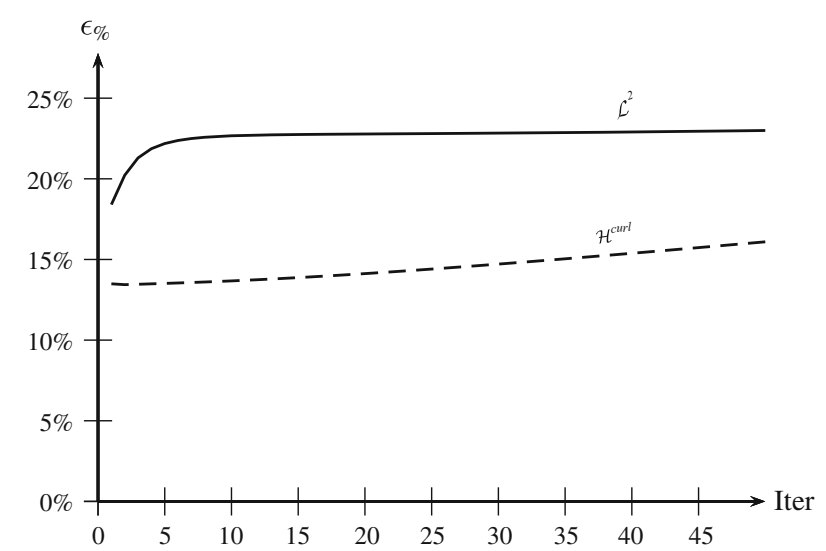

Fig. 12. Relative error for vector potential projection for both $\mathcal{L}^{2}$ and $\mathcal{H}^{1}$. Distances are measured using the norm relative to the magnetic induction $\left(\|\cdot\|_{\text {curl } \cdot \text { curl }}\right)$.

Table 12. Values of $\epsilon_{\text {proj.2 }}^{\%}$ : the error between the projected values over the second mesh for the first iteration.

\begin{tabular}{cc}
\hline Projection & Error $\left(\epsilon_{\text {curl.curl }}^{\%}\right)$ \\
\hline $\mathcal{L}^{2}$ & 18.4035 \\
$\mathcal{H}^{\text {curl }}$ & 13.4904 \\
\hline
\end{tabular}

\subsubsection{Conclusions}

Tests show that the projection of the scalar potential, using the distance minimization of the physical field, does not significantly enhance the solution. Results are slightly more accurate for the first three iterations, but tend to be less precise for a higher number of iterations. There is no advantage of using the $\mathcal{H}^{1}$ projection method in this case.

Unlike the node projection, the $\mathcal{H}^{1}$ projection of edge elements greatly improves the values of the magnetic field. This method seems advantageous as the magnetic potential can be transferred minimizing the error on the magnetic field.

\section{Magneto-mechanical coupled systems}

\subsection{Steady-state models}

Studies presented in the previous sections have allowed to determine how to efficiently transfer data from one mesh to another. The following computation relies on mechanical forces projections (Fig. 13). We will apply the present method to a magneto-mechanical example, the case of a magnetic plate attracted by an electromagnet as described in Figure 15. In that study, one of the lateral surfaces is fixed.

The common quantities between the magnetic and mechanical problems are the force density and the displacement field. Both are discretized with the help of node elements. The mesh dedicated to the mechanical model is composed of ten-node-tetrahedra while the magnetic mesh is composed of linear tetrahedra.

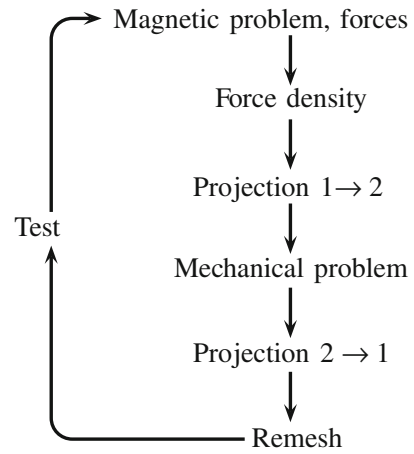

Fig. 13. Detail of the coupling process (steady-state models).

Table 13. Numerical parameters of the static magnetomechanical test case.

\begin{tabular}{ccc} 
& Dimensions & Materials \\
\hline Plate & $20 \mathrm{~cm} \times 20 \mathrm{~cm}$ & $\mu_{r}=1000$, \\
& $h=1 \mathrm{~cm}$ & $E=0.1 \mathrm{~Pa}, \nu=0.3$ \\
Core & $r=2 \mathrm{~cm}, h=20 \mathrm{~cm}$ & $\mu_{r}=1000$, solid part \\
Coil & $r=3 \mathrm{~cm}, h=10 \mathrm{~cm}$ & 1 A total current \\
\hline
\end{tabular}

The properties of the test case are reported in Table 13.

\subsubsection{Source quantities}

Until the plate is not constrained, the only source quantity is the magnetic force density. Although it is a straightforward computation, this problem requires the use of a coupling loop due to his coupled nature.

\subsubsection{Numerical application}

The Young modulus is willingly set to a low value. This ensures a large deformation of the plate and therefore significantly changes the magnetic field distribution. It turns the system into a strongly coupled one. The system is energized at zero time and the current is kept to a constant value over the time. The goal is to compute the final state of the problem.

Because the magnetic state changes with the plate position, an update of the magnetic forces is essential. To achieve this a coupling loop is used as presented in Figure 13.

The coupling process starts with the magnetic resolution over the magnetic mesh (see Fig. 14) which supports linear basis functions. This mesh is composed of the entire electromagnet system and the surrounding air domain. Once the magnetic field distribution is known, nodal force values are computed using the VPP. The next stage computes the force density over the mechanical parts of the first mesh. It ensures that values of forces are suitable to the incoming projection. The projection itself transfers the force density throughout the mechanical mesh (see Fig. 16) and allows the resolution of the mechanical 


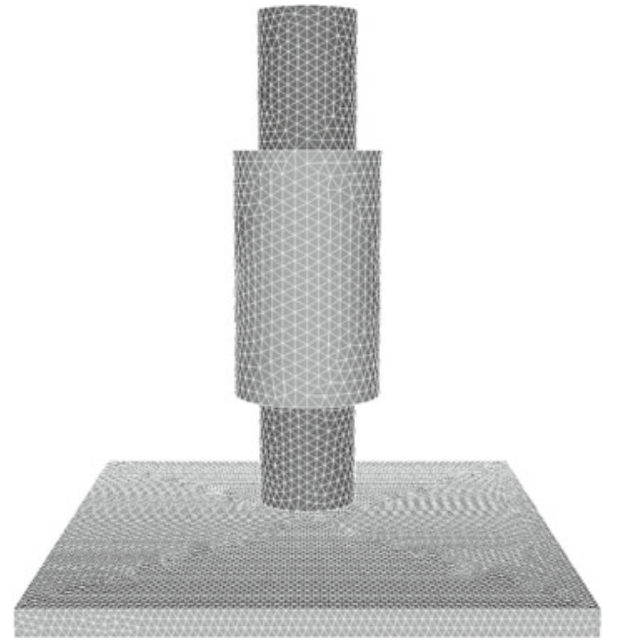

Fig. 14. Mesh of the electromagnet used to solve the magnetic problem (mesh 1). The air box is not represented.

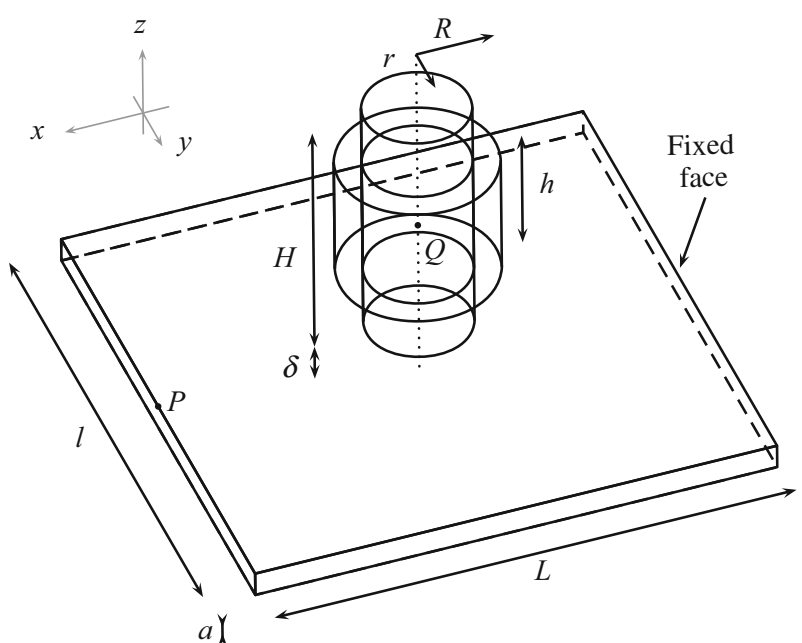

Fig. 15. Detail of the geometry and its parameters. An extra air box is present but not represented.

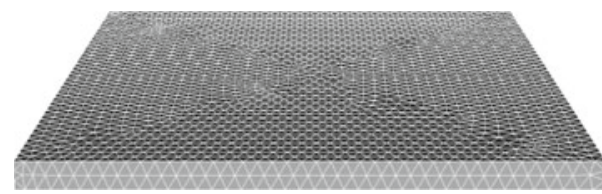

Fig. 16. Mesh of the plate used to solve the mechanical problem (mesh 2). It is fully composed of ten-node-tetrahedra.

problem. A pull-backward projection of the displacement field makes it available over the first mesh. Once the two meshes are updated thanks to the displacement field, another resolution is possible using a modified version of the geometry. The process continues until the equilibrium is obtained [23,24].

The relative displacement between the $(n+1)$ th and the $n$th step is chosen as convergence criterion:

$$
\left\langle 2 \frac{\left\|\mathbf{u}_{n+1}-\mathbf{u}_{n}\right\|}{\left\|\mathbf{u}_{n+1}+\mathbf{u}_{n}\right\|}\right\rangle \leq \epsilon .
$$

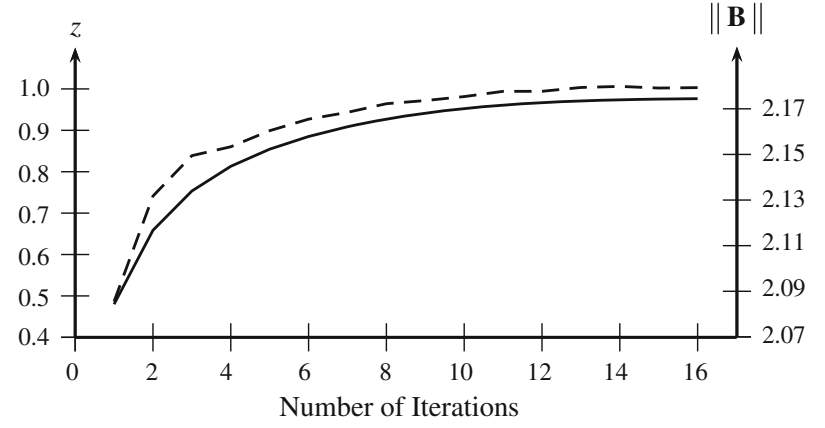

Fig. 17. Vertical displacement of the point $P$, see Figure 15 . Dashed: $\mathbf{B}$ field norm at center of the iron core.

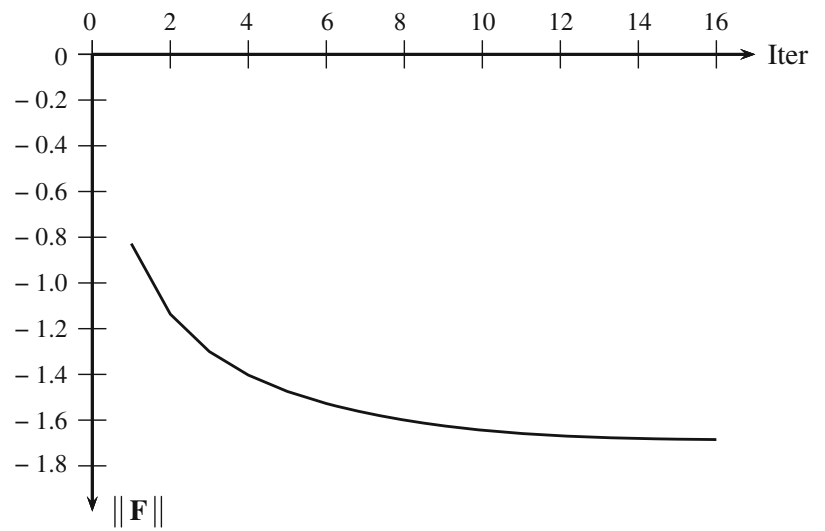

Fig. 18. $z$-component of total force applied to the iron core.

$\epsilon$ is a user predefined value - set to $10^{-4}$ here - which reflects the required accuracy.

The deformation of the plate has noticeable influence on the magnetic field value. Figure 17 shows that $\mathbf{B}$ field norm at the center of the core varies up to $10 \%$ of the final value. ${ }^{11}$

The displacement of the point $P$ has a more understandable variation. The more the plate gets close to the iron core the stronger the forces are. Consequently, the plate is even more attracted. A simple direct computation chain will give a displacement which is twice as low as the final computed value (Fig. 17 step 1 compared to the final value).

At the same time, the total value of the force applied to the iron core is available (see Fig. 18). The value varies according to the evolution of the magnetic field.

The initial and final state are given in Figure 19.

\subsection{Coupling strategies}

The previous subsection gives an overview of a coupled system solved with the help of force density projection.

This strategy is not the only one available. One can also transfer magnetic potentials or magnetic fields. We still consider the displacement field as a return value issued from the mechanical problem. The present test in-

\footnotetext{
11 In this example the air gap remains large and does not allow the field to vary more widely.
} 
(a)

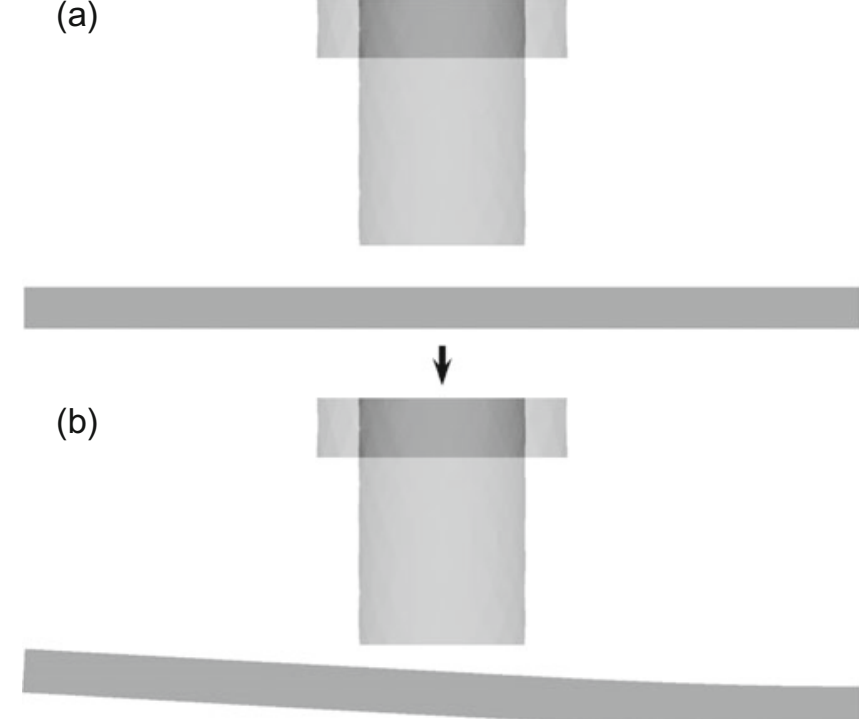

Fig. 19. Plate at (a) initial and (b) final states. Displacement is at scale.

tends to line up the best coupling strategy. Among the possible projected values (the force density, the magnetic potential or the magnetic field) we have tried to determine which one produces the most accurate mechanical values.

To produce an unbiased comparison between these methods, two test-cases have been used. For each case the analytical solution is computed using the second mesh and is taken as reference. Numerical computation of the magnetic model is performed using the first mesh. From this model three coupling strategies are tested using the magnetic potential, the magnetic field (magnetic induction) or the force density. Then the force density and the nodal values of forces are numerically evaluated over the second mesh. The accuracy criterion is defined by the $\mathcal{L}^{2}$ norm of the error between the analytical solution and the numerically computed one.

\subsubsection{Detail of the studied cases}

First example. The cube represents a portion of an infinitely long bar with a uniform current density within it. The domain external to the bar is not modeled as we ensure that the normal component of the field is null over the lateral surfaces. The present cube has $1 \mathrm{~m}$ edge length and the current density (directed along the bar) is equal to $1 \mathrm{~A} / \mathrm{m}^{2}$. In the present case the magnetic permeability has no influence on the field distribution and is arbitrarily set to $\mu_{0}$.

The analytical solution is given by a series:

$$
\boldsymbol{B}\left\{\begin{array}{l}
\frac{16 J \mu_{0} l}{\pi^{3}} \sum_{n} \sum_{p} \frac{\sin \frac{(2 n+1) \pi x}{l} \cos \frac{(2 p+1) \pi y}{l}}{(2 n+1)\left[(2 n+1)^{2}+(2 p+1)^{2}\right]}, \\
\frac{16 J \mu_{0} l}{\pi^{3}} \sum_{n} \sum_{p} \frac{\cos \frac{(2 n+1) \pi x}{l} \sin \frac{(2 p+1) \pi y}{l}}{(2 n+1)\left[(2 p+1)^{2}+(2 p+1)^{2}\right]},
\end{array}\right.
$$

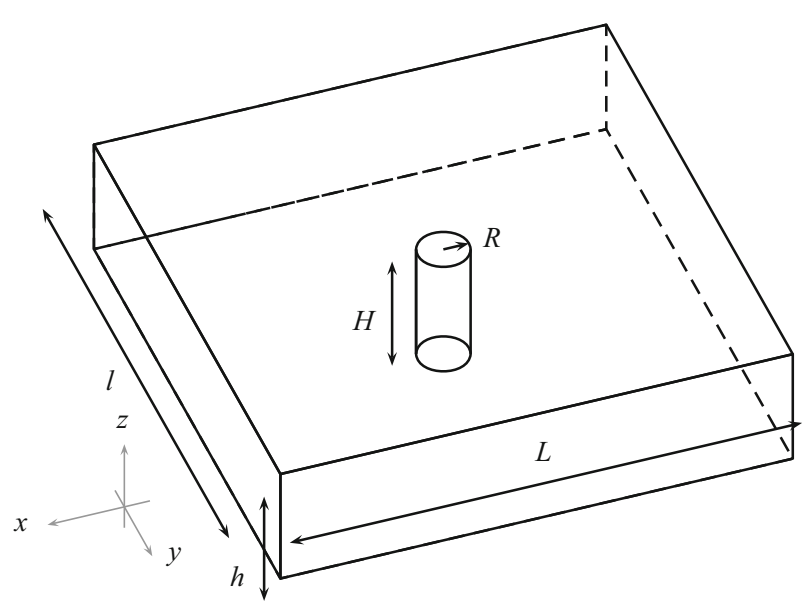

Fig. 20. Detail of the geometry used for the second test case.

for which the first 10000 terms were computed.

First mesh: The mesh is composed of 43000 linear tetrahedra. In this example we have tested both the electric and the magnetic formulations. The first one uses the magnetic vector potential $\mathbf{A}$ and the boundary condition is $\boldsymbol{A} \times n=0$. In the second case, the scalar magnetic potential $\Omega$, for which the Newmann boundary condition $\partial \Omega / \partial n=0$, is imposed and led to the drop of the boundary integral.

The VPP is then applied and using respectively the magnetic energy and the magnetic co-energy. It produces nodal values of forces from which force density is computed using equation (22).

Second mesh: The second mesh is composed of linear tetrahedra. 13000 elements are considered thus the mesh is roughly four times less coarse than the first one. There is no geometrical difference between the "linear" mesh and the "quadratic" one. Discretization nodes are numerically added in the latter one.

The test consists in computing the error committed on forces over the second mesh. Numerical values of forces are successively computed using the projected field among the force density, magnetic field and magnetic potential. The reference solution is computed, over the second mesh, using the analytical solution.

Second example. This case consists in a copper cylinder carrying a uniform current density [25] as represented in Figure 20 . The $\mathbf{B} \times n=0$ boundary condition is still used, and the same set of tests is performed. The main difference lies in a full computation of the mechanical model. The final criterion is the strain value at the center of the copper core.

The modeled wire has $r=1 \mathrm{~mm}$ radius and is made with common copper $(E=120 \mathrm{Gpa}$ and $\nu=0.3)$. The total current is $I=1 \mathrm{~A}$. The analytical value of the strain along the radial axis is given by:

$$
\sigma_{r r}(r)=\frac{-\mu_{0} I^{2}}{16 \pi^{2} R^{2}} \frac{(3-2 \nu)}{(1-\nu)}\left(1-\frac{r^{2}}{R^{2}}\right) .
$$




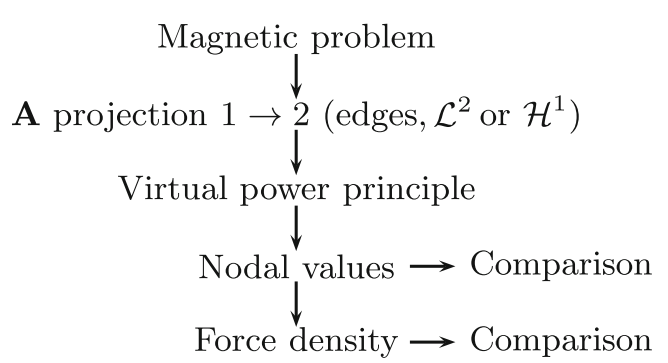

Fig. 21. Details of the stages used for the weak coupling using the magnetic vector potential $\mathbf{A}$.

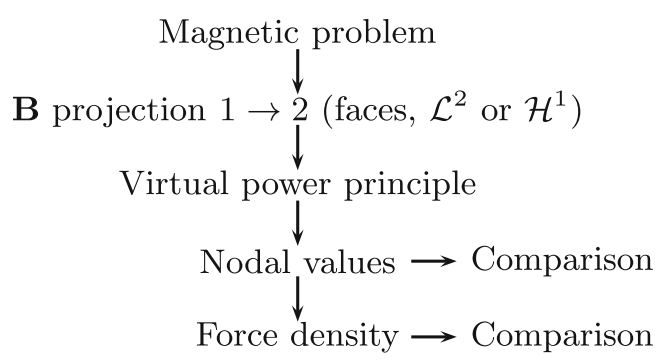

Fig. 22. Weak coupling using B field.

The magnetic mesh is composed of 300000 linear elements. Depending on the method 20000 four-nodetetrahedra or ten-node-tetrahedra compose the second mesh. The air part is withdrawn during the mechanical stage.

A case where $\mathbf{B}$ is transferred through the second mesh was presented in reference [17]. In this study, we also consider the case where the force and the magnetic potential are projected.

\subsubsection{Magnetic potential projection}

This first strategy consists in applying the VPP through the second mesh. In this case one or another magnetic value has to be known. We begin with the use of the projected values of the magnetic potential: $\mathbf{A}$ or $\Omega$ depending on the formulation. The process is presented - for the magnetic vector potential case - in Figure 21, no spatial integration was needed there. The use of the magnetic formulation in $\Omega$ only changes the kind of elements used for the projection.

\subsubsection{Magnetic field projection}

The numerical application of the VPP can also be done using the magnetic field or magnetic induction. Depending on the formulation we have to transfer $\mathbf{B}$ or $\mathbf{H}$. Then the use of the VPP does not change significantly. Figure 22 presents the main stages for the electric formulation. The magnetic formulation case uses $\mathbf{H}$ as state variable and edge elements.

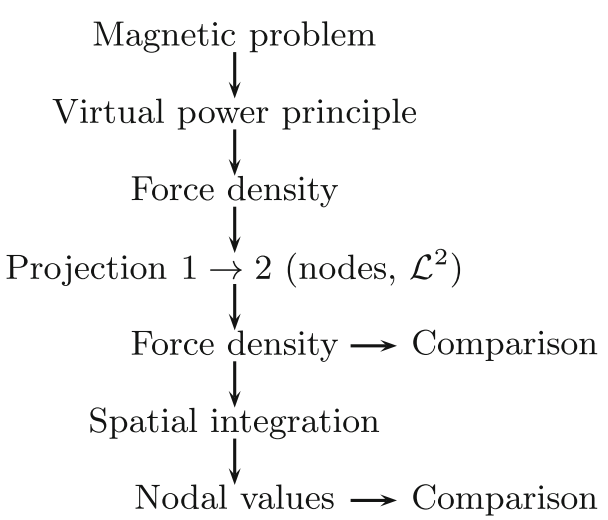

Fig. 23. Main stages used for the coupling using force density. In this case quadratic elements are also considered, the overall process does not change except the projection and the added spatial integration.

\subsubsection{Force density projection}

The main assets of the projection of the force density are the easy implementation of model restriction and the use of quadratic projections. The calculation can be done on moving parts only. This process is used for more complex problems such as the "plate" example (see Fig. 14). In this case VPP is applied on the first mesh. Hence force density is computed. The projection process comes after the application of the VPP. In order to compare the nodal values of forces, an extra spatial integration is needed.

Figure 23 sums up the overall process. The projection stage can either be linear or quadratic and relies on node elements.

\subsubsection{Numerical results, first example}

Computation results. Table 14 presents results obtained for each coupling method. It is subdivided into two parts depending on the formulation (the electric one for the upper part and the magnetic one for the lower part). We focus on the mean relative error per node between the analytical values of the force and the one computed using our method. Results are given in percentage of the analytical value. In order to analyze the possible influence of the strategy on the mechanical problem source data, comparisons for both force density and nodal values were performed.

For each strategy the force computation sequence differs. If magnetic data are transferred nodal forces are computed first, then comes force density. Otherwise force density is computed first, and secondly the spatial integration gives the nodal values. The extra computation stages (spatial integration or force density computation) induce error on the final result. This explains why nodal values are more accurate when transferring the magnetic values. Similarly the force density is closer to the reference solution in the case where forces were transferred.

Figure 24 represents the force density obtained using the nodal projection of the magnetic forces. Figure 25 is 
A.A. Journeaux et al.: Multi-physics problems computation using numerically adapted meshes

Table 14. Accuracy test results for the cube (first example).

\begin{tabular}{cccc}
\hline Projected value & Prod. & Density & Nodal val. \\
\hline Force density (A) & $\mathcal{L}^{2}$ & $1.21 \%$ & $3.78 \%$ \\
Force density quad. (A) & $\mathcal{L}^{2}$ & $0.97 \%$ & $3.12 \%$ \\
Mag. potential, A & $\mathcal{L}^{2}$ & $59.30 \%$ & $52.72 \%$ \\
Mag. potential, A & $\mathcal{H}^{1}$ & $5.25 \%$ & $4.22 \%$ \\
Mag. induction, B & $\mathcal{L}^{2}$ & $5.78 \%$ & $4.62 \%$ \\
Mag. induction, B & $\mathcal{H}^{1}$ & $5.75 \%$ & $4.59 \%$ \\
\hline Mag. potential, $\Omega \& \mathbf{H}_{g}$ & $\mathcal{H}^{1}, \mathcal{L}^{2}$ & $71.47 \%$ & $62.65 \%$ \\
Mag. field, $\mathbf{H}$ & $\mathcal{L}^{2}$ & $15.12 \%$ & $10.80 \%$ \\
Mag. field, $\mathbf{H}$ & $\mathcal{H}^{1}$ & $18.24 \%$ & $12.63 \%$ \\
\hline
\end{tabular}

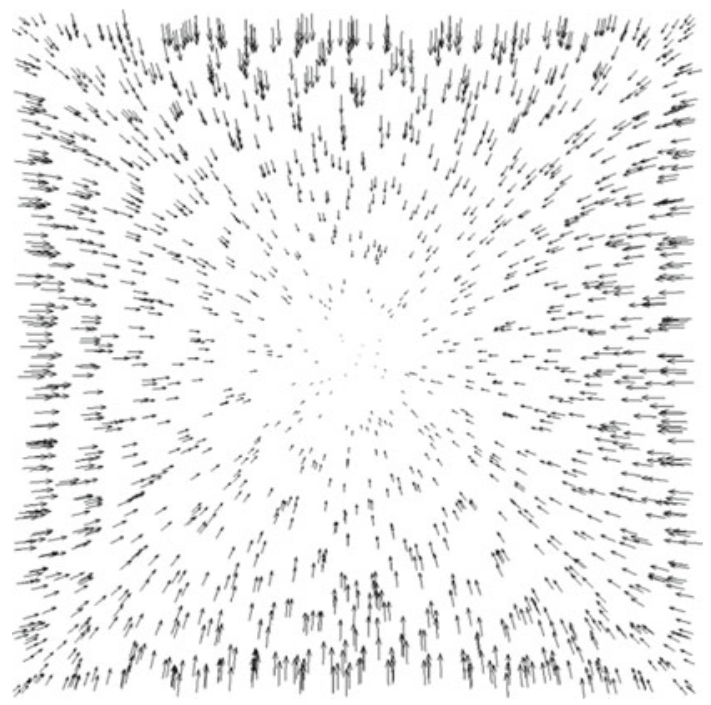

Fig. 24. Projected force density for the cube example.

a plot of the difference between the analytical values and the forces computed using the magnetic potential. High values of the error are located in the corners of the cube.

Comments on results. The use of force density as a common value appears to be the best choice for weak coupling. Force density is the natural source data for the mechanical problem. The use of quadratic basis functions improves the accuracy of the mechanical problem while it does not harden the implementation. Furthermore, it facilitates the reduction of the model: a local renumbering of selected elements divides by 20 the projection time.

However if source data for the mechanical code are nodal force values, the use of $\mathbf{B}$ field is also a good choice. In this case, the use of quadratic elements and spatial restriction are then more difficult.

Results show the interest in the use of the $\mathcal{H}^{1}$ norm for the projection of magnetic potentials. The accuracy of the fields and therefore the one of forces is greatly improved in the $\mathcal{H}^{1}$ case compared to the $\mathcal{L}^{2}$ case. This result high-

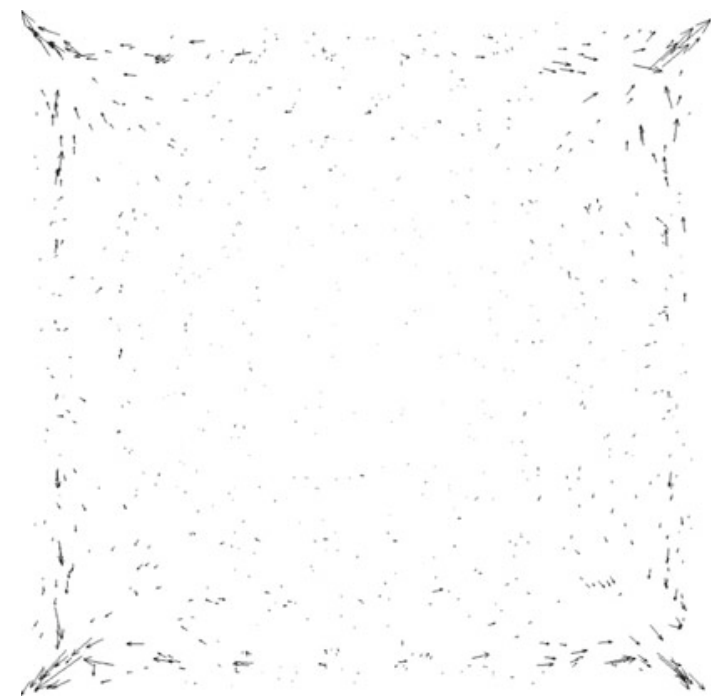

Fig. 25. Difference between projected forces and those stemming from the $\mathcal{H}^{1}$ projection of the magnetic potential. Errors are essentially located around the corners of the cube.

Table 15. Accuracy test results for the magneto-mechanical cylinder (second example).

\begin{tabular}{cccc}
\hline Projected value & Prod. & Value $/ 10^{-3}$ & Relative error \\
\hline Force & $\mathcal{L}^{2}$ & -26.89 & $1.44 \%$ \\
Force quad. & $\mathcal{L}^{2}$ & -27.06 & $0.82 \%$ \\
$\mathbf{A}$ & $\mathcal{L}^{2}$ & -19.33 & $29.15 \%$ \\
$\mathbf{A}$ & $\mathcal{H}^{1}$ & -26.66 & $2.29 \%$ \\
$\mathbf{B}$ & $\mathcal{L}^{2}$ & -26.67 & $2.25 \%$ \\
$\mathbf{B}$ & $\mathcal{H}^{1}$ & -24.45 & $10.39 \%$ \\
\hline Force & $\mathcal{L}^{2}$ & -27.27 & $0.05 \%$ \\
Force quad. & $\mathcal{L}^{2}$ & -27.30 & $0.06 \%$ \\
$\Omega \& \mathbf{H}_{g}$ & $\mathcal{H}^{1}, \mathcal{L}^{2}$ & -11.04 & $59.54 \%$ \\
$\mathbf{H}$ & $\mathcal{L}^{2}$ & -18.13 & $33.55 \%$ \\
$\mathbf{H}$ & $\mathcal{H}^{1}$ & -17.42 & $36.15 \%$ \\
\hline
\end{tabular}

lights the importance of considering the physically meaningful field in the projection process.

\subsubsection{Numerical results, second example}

Numerical computations are presented in Table 15 and are consistent with the results obtained for the first case. Note that the projected quantities are force density, $\mathbf{A}$, $\mathbf{B}, \Omega$ or $\mathbf{H}$ depending on the method and the formulation. This time the only source data for the mechanical model is force density. It implies the numerical prepossessing of the nodal values when magnetic quantities are transferred.

The analytical value for the strain is $\sigma_{r r}(r=0)=$ $-27.284 \times 10^{-3} \mathrm{~Pa}$. A cut plane of the radial related component of the strain is given in Figure 26. 


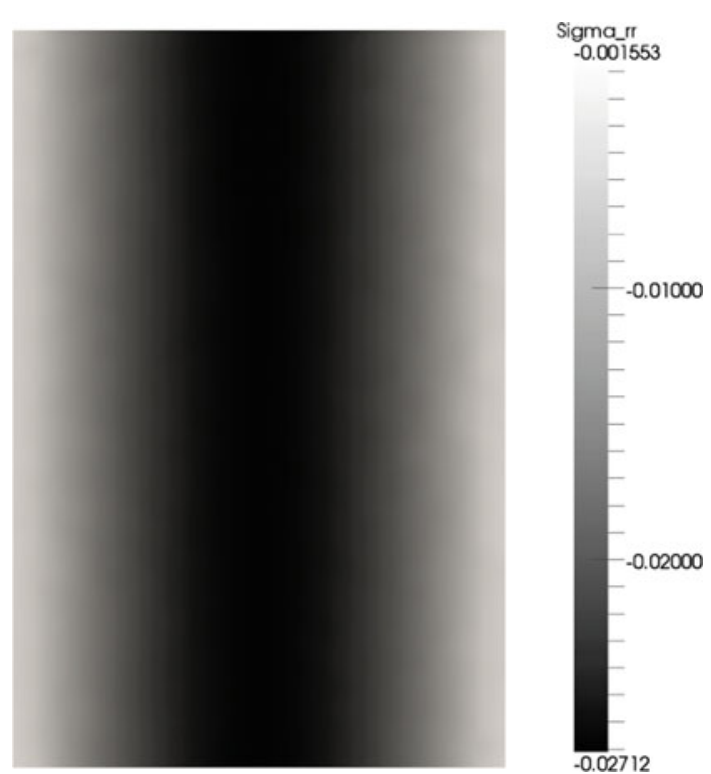

Fig. 26. Strain distribution along the radial axis.

\subsection{Dynamical systems}

\subsubsection{Introduction}

The projection method can also be applied to transient state of magneto-mechanical coupled systems. For the sake of simplicity we now consider mechanically dynamical systems while the magnetic model is still considered in its steady-state form. In other words, displacements are slow enough - considering magnetic field evolution and the conductivity of the materials - to not induce currents that have significant effects on the mechanical evolution.

The study is restricted to the simple case for which the present method is performed for a unique time step. To perform such calculation different methods exist, however their review - which would need a full study - will not be presented. A more thorough analysis of time schemes is presented in reference [26].

\subsubsection{Dynamical magneto-mechanical processes}

We now consider a transient mechanical model: the massmatrix $[M]$ is introduced and time evolution is taken into account using an implicit time discretization method. ${ }^{12}$ To avoid burdening the description of the present method, it is assumed that the time step is small enough to correctly compute the evolution of the process. Obviously more complex time discretization methods can be used.

\subsubsection{Numerical example, the damped plate}

The plate now has a density equal to $\rho=10^{-2} \mathrm{~kg} \mathrm{~m}^{3}$ which is low enough to prevent contact with the iron core.

\footnotetext{
12 We have used a Newmark scheme due to its unconditional stability.
}

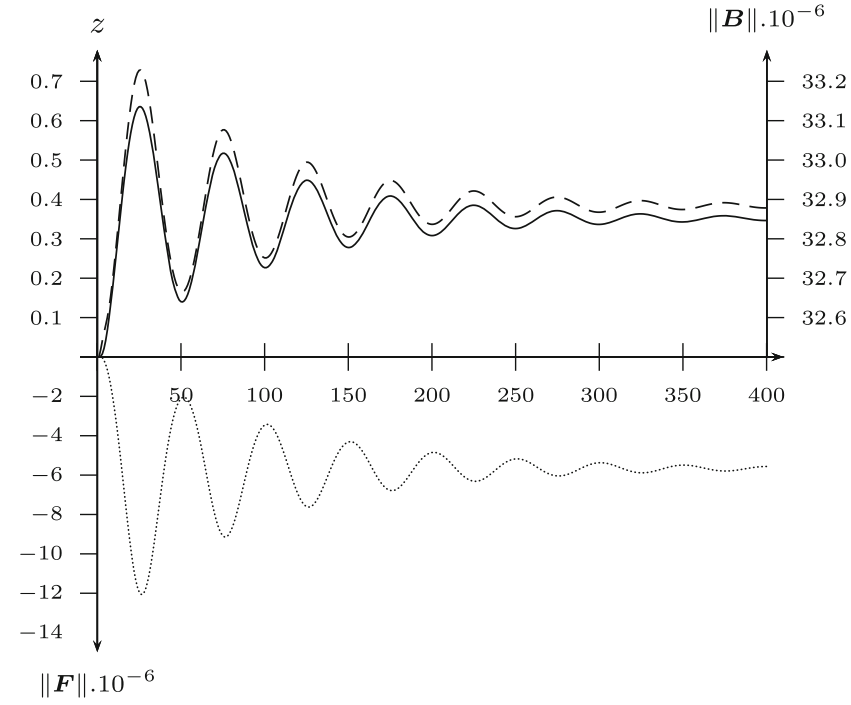

Fig. 27. Solid: vertical displacement of the point $P$ using transient analysis. Dashed: magnetic field norm at the center of the iron core. Dotted: total force, along the vertical direction, applied to the electromagnet.

In order to obtain around 10 oscillations, and despite the lack of significance of such a formula, a Rayleigh damping model is also introduced: the damping matrix is calculated from a linear combination of the mass and stiffness matrices. It leads to the following linear system:

$$
[\mathbf{M}] \partial_{t}^{2}[\mathbf{u}]+[\mathbf{D}] \partial_{t}[\mathbf{u}]+[\mathbf{K}][\mathbf{u}]=[\mathbf{f}]
$$

Results obtained using the weak coupling and field projections are presented in the following part. We have computed the response of the system, initially unenergized, to a current step. Figure 27 presents the vertical displacement of the point $P$ (see Fig. 15) using this transient analysis.

We have also noticed that computed electromagnetic fields - hence forces - are time-dependent bringing out the influence of air gap size. Further analysis shows that the present method gives good results. Different time scheme were studied in reference [26], their accuracy and computation time were compared.

\section{Magneto-thermo-mechanical coupled systems}

The next part discusses the three-physics modeling. Processes used for the magneto-mechanical coupling method are mainly reused. A thermal model is now added to the previous magneto-mechanical model. This time coupling through the constitutive law is involved in the overall process. We deliberately highlight that the present framework also applies to systems coupled by constitutive laws, and not to solely external loads.

The added physics is solved in its own frame; a supplementary mesh is used for it. It also uses two more projections processes. 


\subsection{Process overview}

Previous work relies on magnetostatics: the only currents that may exist belong to inductors and are considered as sources. Joule losses can be obtained by specifying conductivity to the domain. Their values are given by $s=|\boldsymbol{J}|^{2} / \sigma$. This is typically what is used in the example of Section 4.2 .

The magnetic model is revised to become a transient one, and the eddy-current model is then used. This model, which merely represents Maxwell's equations, is characterized by the drop of the displacement current term $\left(\partial_{t} \boldsymbol{D}\right)$. For each formulation an additional potential is required. It leads to the so-called "electric" and "magnetic" formulations, for which equations are respectively:

$$
\begin{array}{r}
\operatorname{rot}\left(\frac{1}{\nu} \operatorname{rot} \mathbf{A}\right)+\sigma\left(\partial_{t} \mathbf{A}+\operatorname{grad} \phi\right)=\mathbf{J}_{g}, \\
\operatorname{curl} \frac{1}{\sigma} \operatorname{curl} \mathbf{T}+\mu \partial_{t}(\mathbf{T}-\operatorname{grad} \Omega)= \\
-\operatorname{curl} \frac{1}{\sigma} \operatorname{curl} \mathbf{H}_{g}-\mu \partial_{t} \mathbf{H}_{g},
\end{array}
$$

where $\phi$ is the electric scalar potential and $\mathbf{E}=-\partial_{t} \mathbf{A}-$ $\operatorname{grad} \phi . T$ is the electric vector potential, and $\mathbf{E}=\operatorname{rot} T / \sigma$.

Regardless of the used formulation, a prerequisite to the mechanical problem is the full determination of the temperature distribution. To do this, two additional stages are necessary:

- determination of the Joule losses using the equation (23);

- thermal problem computation using transferred sources.

\subsubsection{Transferred data}

Joule losses denoted by $s$ are computed with the help of the magnetic basis functions. Moreover, losses are scalar values, constant by element and are discretized using volume elements.

As we use a specific mesh for the thermal problem, we have to transfer this loss density. To do this we use an element to element projection method, which is conservative and defined by:

$$
\int_{\mathcal{K}_{t_{i}}} s_{t}=\int_{\mathcal{K}_{t_{i}}} s_{s},
$$

where $\mathcal{K}_{t_{i}}$ denotes the $i$ th element of the mesh associated to the thermal problem (target one). As the volume elements basis functions is written $w^{i}=1 / \operatorname{vol}\left(\mathcal{K}_{i}\right)$ - which is constant by element - multiplying equation (53) by $\psi$ chosen among the $w_{t_{j}}$ one gets:

$$
\left\langle s_{t}-s_{s}, \psi\right\rangle=0 \forall \psi \in\left\{w_{t_{j}}\right\} .
$$

We do recover the method used in Section 1.5. This statement remains important: projection of Joule losses using volume elements keeps the total heat power despite changes in the distribution.

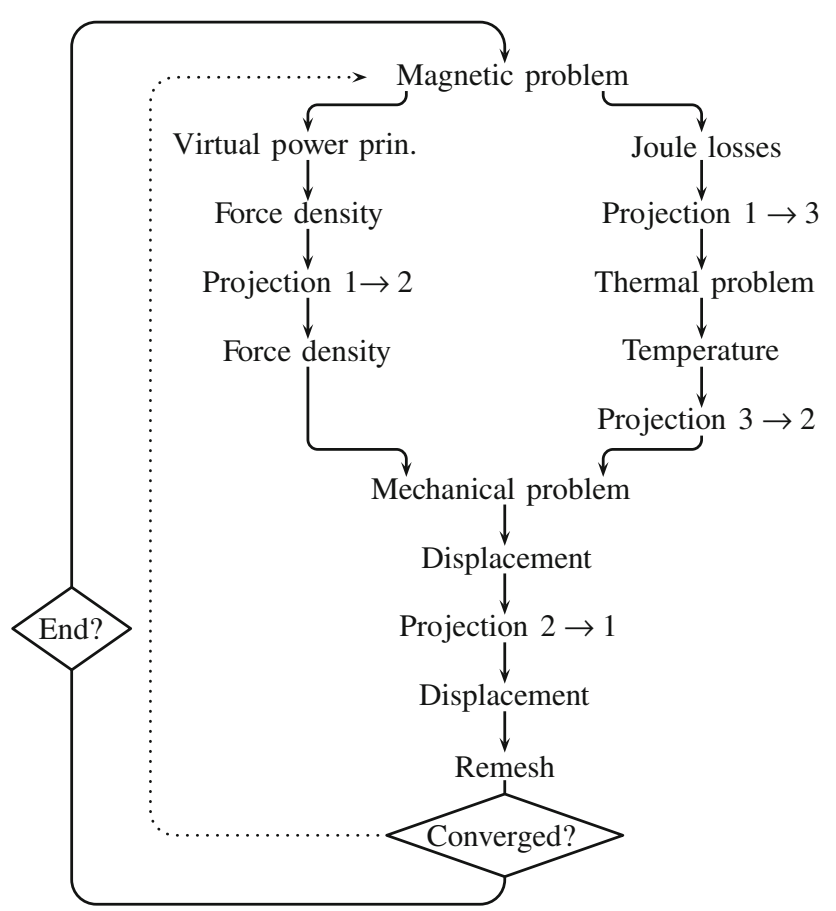

Fig. 28. Magneto-thermo-mechanical coupling process stages. We denote by 1 the magnetic mesh, by 2 the mechanical mesh and by 3 the thermal one.

By the resolution of the thermal problem, the temperature distribution can be determined. This field is discretized using node elements and has to be transferred to the mechanical model.

\subsubsection{Detail of the stages}

Three different meshes are used: one per phenomenon. As a consequence we have to deal with three kinds of projections.

As presented previously, it is necessary to process nodal forces in order to get the force density which is compliant with projection. Magneto-mechanical projection involves forces and uses the $\mathcal{L}^{2}$ node-to-node projection in its vectorial form. This first kind of projection involves magnetic and mechanical parts of the process, and defines force load applied to the mechanical media.

Magnetic model also provides ohmic losses present in conducting parts. The second kind of data transfer relies on element-to-element projection and makes possible the determination of Joule losses over the thermal mesh starting from the ones on the magnetic mesh.

In order to correctly determine the mechanical behavior law, this distribution also has to be determined within the mechanical parts. As the temperature is discretized using node elements, an $\mathcal{L}^{2}$ node-to-node elements projection stage is used.

At this point, all data required by the mechanical problem are available through the mechanical parts. The displacement field can be determined through the resolution 


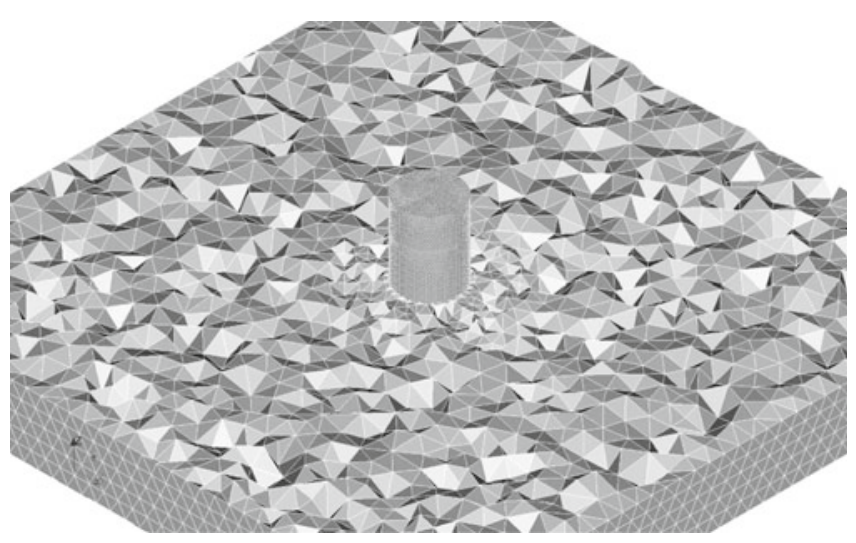

Fig. 29. Illustration of the geometry after meshing.

of the mechanical problem, thus paving the way for the geometrical update.

Figure 28 is a summary of the flowchart of the overall problem. In order to cut computational time, as thermal part of the process is independent of the force density projection, these two operations can be performed simultaneously.

\subsection{Comparison with an analytical model}

\subsubsection{Test-case description}

Similarly to the magneto-mechanical case, an electric heated cylindrical wire is considered. The geometry is the same as in Section 3.2.1 and is presented in Figure 20. More precisely the test-case geometry is unchanged but the combination of the physical models differs. The magnetic behavior is roughly the same, namely a uniform current density belonging to the conductor. All parts behave like $\mu_{r}=1$ materials (e.g., air or copper). Moreover, we take into account the copper's conductivity $\sigma$. As current density is imposed, the overall magnetic field distribution is unchanged. The addition of a conductivity allows the computation of Joule losses using equation (23).

An example of the mesh used for the computation is available in Figure 29. The upper half of the air part was removed, thus unveiling the mesh of the wire. The mechanical behavior law is now defined by equation (24): temperature is issued from the thermal computation while force density is computed using the VPP, "density" transform, and projection chain.

As we wish to measure the accuracy of the coupled system, the worst case must be considered. To do this, we ensure that every linkage produces a deformation of the same order. A wise set of parameters is then used despite the loss of the physical significance.

As boundary condition, the Newton's law of cooling ( $h$ coefficient) is applied to the lateral surface of the conductor. As described in Section 1.3 the field of temperature is computed using a linear model of conduction (coefficients $\rho C_{p}$ and $\left.k\right)$.

All used parameters are reported in Table 16 where $R$ is the radius of the cylinder, $I$ is the total current within
Table 16. Model parameter values for the heated cylinder.

\begin{tabular}{rlrl}
\hline$\mu$ & $=\mu_{0}$ & $I$ & $=1 \mathrm{~A}$ \\
$E$ & $=1 \mathrm{~Pa}$ & $\nu$ & $=0.3$ \\
$k$ & $=10^{-5} \mathrm{~W} \mathrm{~m}^{-1} \mathrm{~K}^{-1}$ & $\sigma_{m}$ & $=10^{7} \mathrm{~S} \mathrm{~m}^{-1}$ \\
$\alpha$ & $=5 \times 10^{-4} \mathrm{~K}^{-1}$ & $h$ & $=2 \times 10^{-1} \mathrm{~W} \mathrm{~m}^{-2} \mathrm{~K}$ \\
$T_{\text {ext }}$ & $=20^{\circ} \mathrm{C}$ & $R$ & $=1 \mathrm{~mm}$ \\
\hline
\end{tabular}

it. $\mu_{0}$ is the permeability of vacuum common to air and copper materials. $E$ and $\nu$ are respectively Young modulus and Poisson's ratio which parameterizes the mechanical behavior law.

The magnetic mesh includes an air box and is composed of 400000 linear tetrahedra. The mechanical mesh is supported by the cylinder shape only and is composed of 23600 quadratic tetrahedra. The thermal mesh is composed of 21200 tetrahedra.

\subsubsection{Numerical results}

Analytical model computation. The accuracy test consists in computing the radial component of strain at the center of the cylinder. An analytical computation has been performed in reference [27] leading to:

$$
\begin{aligned}
& \sigma_{r r}= \\
& \frac{-\mu_{0} I^{2}}{16 \pi^{2} R^{2}}\left(\frac{\nu}{3-2 \nu}+\frac{\alpha E}{\mu_{0} k \sigma_{m}(1-\nu)}\right)\left(1-\frac{r^{2}}{R^{2}}\right),
\end{aligned}
$$

which takes a negative value as forces tend to compress the cylinder. The temperature distribution decreases as $r^{2}$ and the heat power balance gives the expression of the exterior surface temperature $T_{\text {sur }}$ :

$$
T_{\text {sur }}=T_{\text {ext }}+\frac{I^{2}}{2 \pi^{2} h \sigma R^{3}} .
$$

Using the present set of parameter values, and applying above formulae, one obtains: $\sigma_{r r}=-34.82 \times 10^{-3} \mathrm{~Pa}$ and $T_{l}=24.22{ }^{\circ} \mathrm{C}$ for the lateral surface temperature. These results are in agreement with the one presented in Section 3.2.6.

Numerical application. The method was first tested on a single-mesh method for which no projections were involved. We have then used, for the copper cylinder, the same mesh for the three models. The magnetic mesh only differs as an air box is required. The aim is to check the full integrity of the model and to perform a first assessment of the exactness of the process. We have used three different meshes adapted to each physical model. The results issued from the projection method have to be compared to the one-mesh process (see Tab. 17).

In order to obtain similar behavior, the mesh used for the one-mesh method is a combination of the air part of the magnetic mesh and the cylindrical part of the mechanical one. The use of a single mesh avoids resorting to 
Table 17. Accuracy test results for the magneto-thermomechanical test case.

\begin{tabular}{ccccc}
\hline Projected value & Prod. & Type & $\begin{array}{c}\text { Value } \\
10^{-3}\end{array}$ & $\begin{array}{c}\text { Rel. error } \\
(\%)\end{array}$ \\
\hline None (single mesh) & - & $T_{l}$ & 24.21 & 0.04 \\
None (single mesh) & - & $\sigma_{r r}$ & 34.58 & 0.69 \\
\hline Temperature (A) & $\mathcal{L}^{2}$ & $T_{l}$ & 24.28 & 0.25 \\
\hline Forces (A) & $\mathcal{L}^{2}$ & $\sigma_{r r}$ & 34.78 & 0.11 \\
$\mathbf{A}$ & $\mathcal{L}^{2}$ & $\sigma_{r r}$ & 27.42 & 21.25 \\
$\mathbf{A}$ & $\mathcal{H}^{1}$ & $\sigma_{r r}$ & 34.57 & 0.72 \\
$\mathbf{B}$ & $\mathcal{L}^{2}$ & $\sigma_{r r}$ & 34.56 & 0.75 \\
$\mathbf{B}$ & $\mathcal{H}^{1}$ & $\sigma_{r r}$ & 32.89 & 5.54 \\
\hline Temperature $(\Omega)$ & $\mathcal{L}^{2}$ & $T_{l}$ & 24.28 & 0.25 \\
\hline Forces $(\Omega)$ & $\mathcal{L}^{2}$ & $\sigma_{r r}$ & 35.01 & 0.55 \\
$\Omega$ \& H & $\mathcal{H}^{1}, \mathcal{L}^{2}$ & $\sigma_{r r}$ & 19.24 & 44.74 \\
$\mathbf{H}$ & $\mathcal{L}^{2}$ & $\sigma_{r r}$ & 25.28 & 27.40 \\
$\mathbf{B}$ & $\mathcal{H}^{1}$ & $\sigma_{r r}$ & 22.92 & 34.18 \\
\hline
\end{tabular}

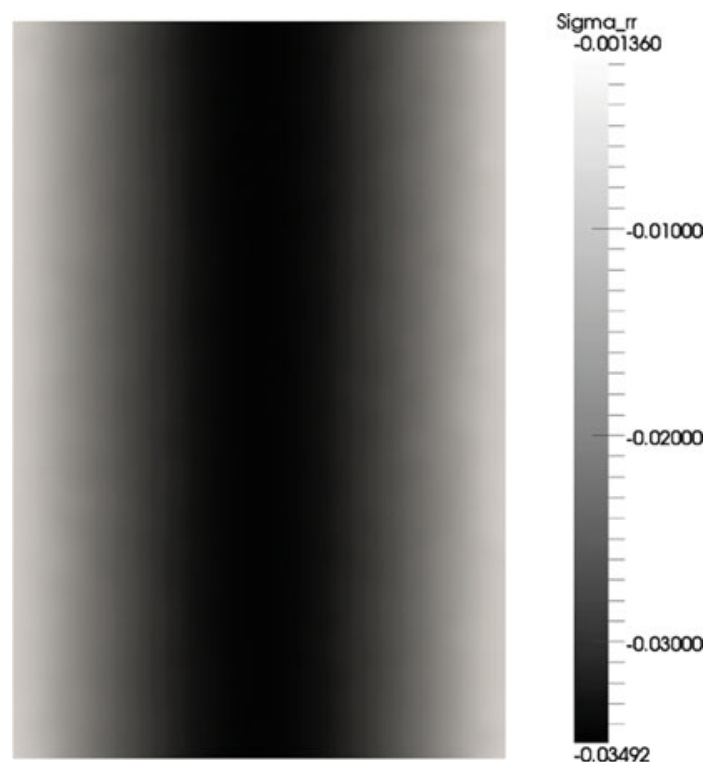

Fig. 30. Strain distribution along the radial axis.

projections but is highly disadvantageous in terms of computational cost. Nevertheless, the complexity introduced by the projection stages does not significantly decrease the final accuracy.

Figures 30 and 31 present numerical results obtained using the projection method. Figure 30 is a plot of the radial component of the strain tensor: values are negative as forces tend to tighten the core. As the heat is evacuated through the lateral surfaces, the highest temperatures are located at the center of the wire (Fig. 31). Because of the circular invariance of the geometry, values only depend on the radial position.

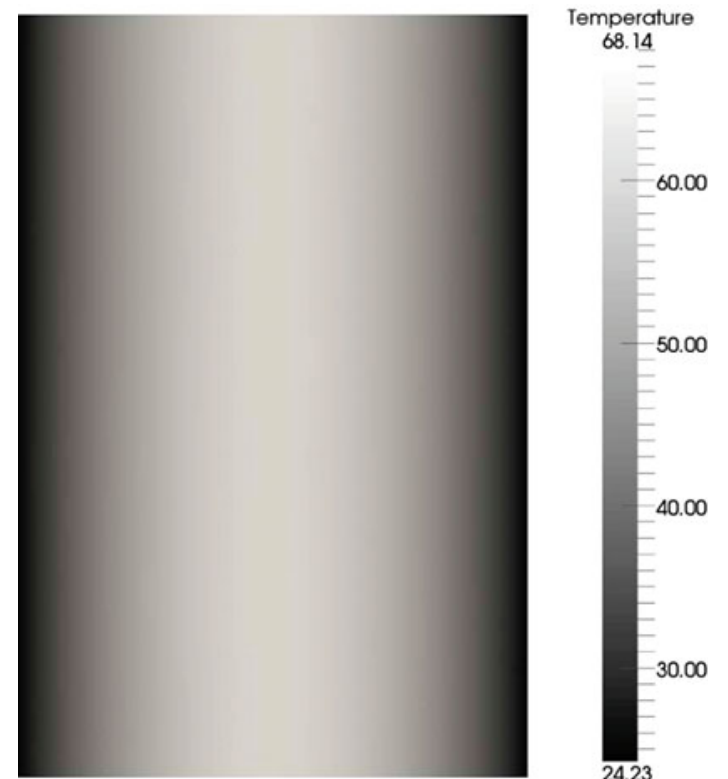

Fig. 31. Temperature distribution along the radial axis.

\subsection{Dynamical system example}

The previous analytical test does not involve the complex re-meshing process. Regrettably we have not found an analytical solution of a transient magneto-thermo-mechanical system. The further computation does not intend to produce a performance test. It is more a demonstration of feasibility of such a computation than a benchmark.

The ferromagnetic damped plate actuated by an electromagnet provides a highly magneto-mechanical coupled system. For consistency's sake we have reused the previous model, to which we have introduced the missing magnetothermal and thermo-mechanical couplings.

The magneto-thermal coupling which is commonly encountered is heating due to eddy currents. We now go on with dynamic magnetic systems containing conductive parts. The introduction of the missing thermo-mechanical coupling only remains. In an effort to produce a system for which the shape highly depends on the temperature, we focus on bimetallic plates.

\subsubsection{Setup descriptions: the damped ferromagnetic bimetallic plate}

The structure of the system is roughly the same as in Section 3.3.3. Dimensions and physical properties of the iron core and the copper coil do not change and are still considered as fixed parts. Except from its width the plate is not modified and is composed of ferromagnetic materials. In order to get induced currents within the plate, we now consider the material as conductive. The bimetallic effect is obtained by taking a nonzero coefficient of thermal expansion. The two parts of the bimetallic strip - the upper and lower half of the plate - have respectively a coefficient of opposite sign. 


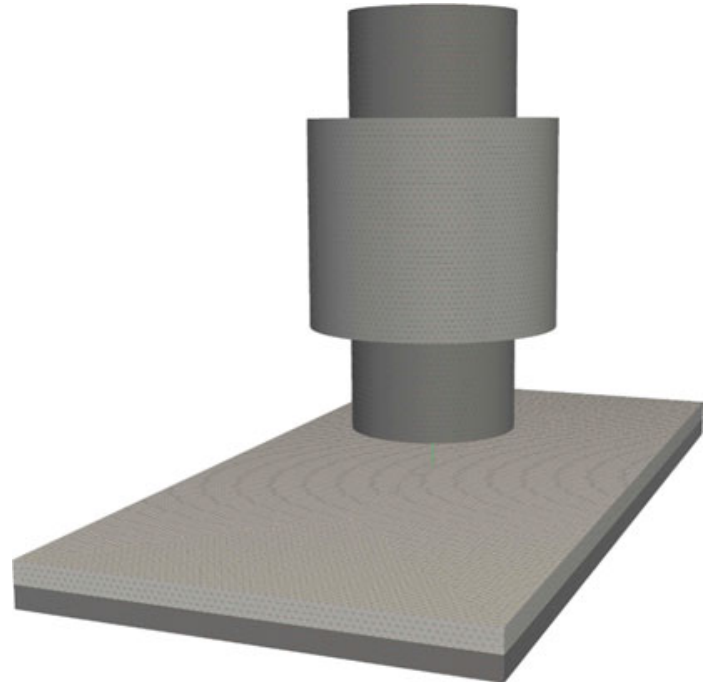

Fig. 32. Details of the considered electromagnet and strip. Air not represented.

The source current varies sinusoidally through the time. The frequency of this source is supposed sufficiently high to enable the approximation of the force - through a mechanical time step - by its average value.

Figure 33 is a sketch of the current geometry. Meshes are then defined by:

- The "magnetic" mesh consisting of: the iron core, the coil, the plate and the surrounding air. This mesh is composed of 378000 of the so-called "four-node" linear tetrahedra. Mesh characteristic size is significantly lower than the two following ones. This mesh, for which the air part was withdrawn, is presented in Figure 32.

- The "thermal" mesh is only related to the plate as we focused on the temperature of the bimetallic strip only. It consists in 127000 linear tetrahedra. At this point we do not distinguish the two parts of the strip either for the "magnetic" mesh or for the "thermal" one.

- The remaining mesh is the "mechanical" one. As well as for the thermal mesh, the mechanical domain is limited to the plate only. As described before we have used "ten-node-tetrahedra" which support quadratic basis functions. This kind of elements suit well the mechanical formulation.

Every parameters involved in the model is gathered in Table 18. One must carefully keep in mind that some parameters may not be physically meaningful. Like it has been done for the analytical case, we have tried to obtain the "strongest" coupled case. Namely it is the case where none of the three physical models entirely rules the evolution of the system. Every linkage between sub-problems has its importance and thus highly interferes with the other ones.

As shown in Figure 33 the bimetallic trip is composed of two sub-shapes defined by their length $L$ and width $l$. Their respective height is $a$. The iron core and its surrounding copper coil are defined using two concentric cylinders which have respectively $H$ and $h$ height, radii are $r$ and $R$. The initial air gap is defined by $\delta$ which is the distance between the bottom of the core and the top of the

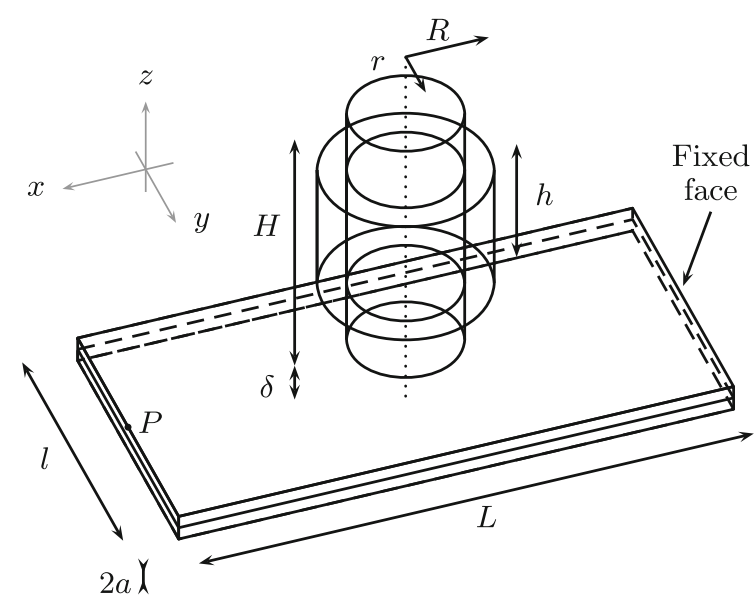

Fig. 33. Sketch of the geometry of the bimetallic strip.

Table 18. Lengths defining the system. Distances are given in meters.

\begin{tabular}{cccc}
\hline$L$ & 20 & $l$ & $10 \mathrm{~A}$ \\
$a$ & $0.5 \mathrm{Mpa}$ & $\delta$ & 1 \\
$h$ & 5 & $H$ & 10 \\
$r$ & 2 & $R$ & 3 \\
$L_{\text {air }}$ & 60 & & \\
\hline
\end{tabular}

strip. An additional cube - which is $L_{\text {air }}$ edge length - is defined and represents the added air used in the magnetic domain.

Materials properties, using the same notation as in Section 4.2 , can be found in Table 19 . We recall that the damping matrix is computed using the linear combination: $[M]=\alpha_{d}[K]+\beta_{d}[M]$.

In the present case $\mu$ stands for the relative permeability of every ferromagnetic part: the iron core and the strip. Every other part such as air or coil behaves like vacuum $\left(\mu_{0}\right)$. Each slice of the strip has different coefficient of thermal expansion: the upper half has $\alpha_{\text {upper }}=\|\alpha\|$ while the lower one has $\alpha_{\text {lower }}=-\|\alpha\|$.

The system is initially considered at its extinct state: no current within the coil and no motion. The temperature is set to $20{ }^{\circ} \mathrm{C}$ at every point of the domain. The modeling starts with the energizing of the coil. The RMS value of the current is maintained constant from the beginning up to the 1000th time step. The coil is then switched off

Table 19. Physical properties values for the "bimetallic strip" test-case.

\begin{tabular}{cccc}
\hline$\mu$ & 1000 & $I$ & $1 \mathrm{~A}$ \\
$E$ & $1 \mathrm{MPa}$ & $\nu$ & 0.3 \\
$\rho$ & $1 \mathrm{~kg} \mathrm{~m}^{-3}$ & $\rho \times C p$ & $3.3 \times 10^{-3} \mathrm{~J} \mathrm{~K}^{-1} \mathrm{~m}^{-3}$ \\
$\|\alpha\|$ & $2 \times 10^{-6} \mathrm{~K}^{-1}$ & $h$ & $2 \times 10^{-1} \mathrm{~W} \mathrm{~m}^{-2} \mathrm{~K}$ \\
$T_{\text {ext }}$ & $20{ }^{\circ} \mathrm{C}$ & $k$ & $10^{-5} \mathrm{~W} \mathrm{~m}^{-1} \mathrm{~K}^{-1}$ \\
$\alpha_{d}$ & 1 & $\beta_{d}$ & 1 \\
\hline
\end{tabular}




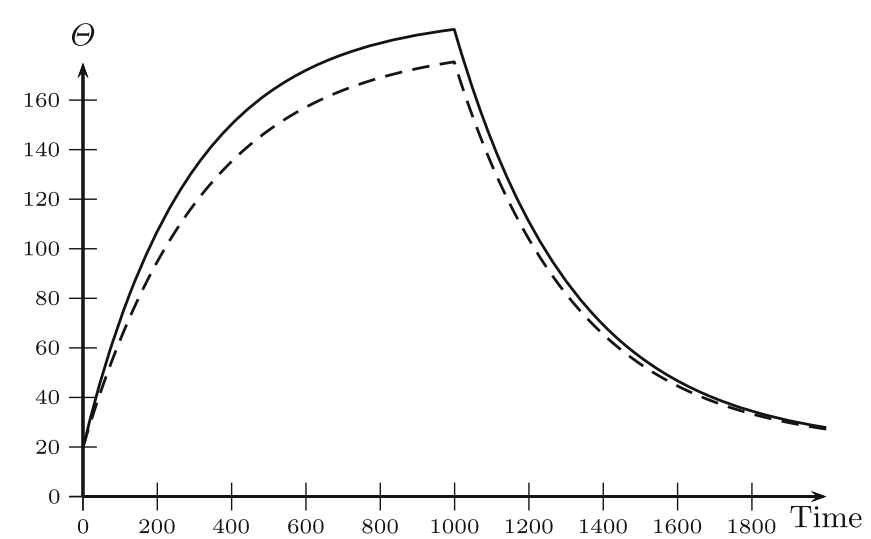

Fig. 34. Solid: temperature at the center of the strip (with motion). Dashed: plot of the temperature for the non-moving case.

and the system moves freely up to its equilibrium point. From the 1001th time step until the end of the computation, we actually model a thermo-mechanical system as no magnetic field is considered.

\subsubsection{Numerical results}

The most remarkable point is the variation of the temperature of the center of the strip over the time. Figure 34 presents the variation of the temperature over the time for both the present case and the reference case. In order to point out that the strip shape modification of the plate has influence on the induction heating, we plot the curves with (solid line) and without (dashed line) motion.

We denote by reference the purely magneto-thermal case where no deformation was considered. As the air gap decreases the magnetic field rises and hence the Joule losses rise too. One can notice that, in the present case, the committed error on the maximum values goes up to $15{ }^{\circ} \mathrm{C}$. It represents $10 \%$ of the total overheating. This statement is one of the signs that proves the need to take into account the three models together.

Figure 35, as in Section 3.3.3, represents the vertical variation of the point $P$ which is the middle point of the upper edge of a lateral face.

As shown in Figure 35 at least four phases clearly appear during the system's evolution:

- The first transitional state (1) is due to the mechanical stroke subsequent to the apparition of magnetic forces. Oscillations extensively arise from the inertia of the plate (the mass density $\rho$ is no longer neglected). They are gradually vanishing as the mechanical problem is damped. The authors intentionally set the value of damping factors $\alpha_{d}$ and $\gamma_{d}$ to get a short mechanical transitional state compared to the thermal one;

- As suggested in the previous point phase (2) shows the bend of the bimetallic strip due to the increasing temperature. Despite the relative similarity to the constant heating case, the shape of the curve has a complex evolution involving the variation of $s$ (the heating

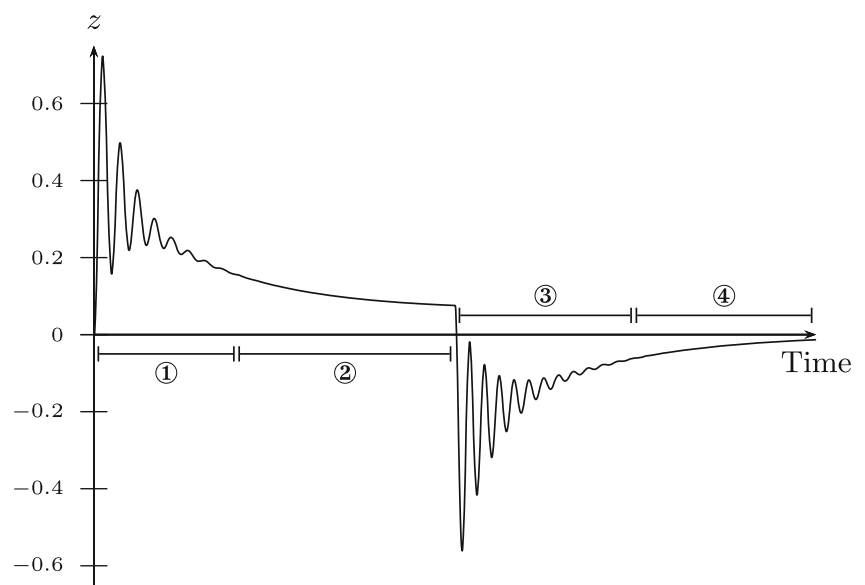

Fig. 35. Plot of the vertical displacement over the time (point $P$, see Fig. 33)

sources). We almost awaited the equilibrium state of the energized system before switching the coil off.

- During the next two stages, the system reduces to a thermo-mechanical one. Phase (3) reflects the mechanical transitional state due to the sudden release of magnetic forces. The strip spontaneously stretches toward the opposite side as materials are still hot.

- Finally the strip tends to recover its natural state owing to the imposed boundary conditions that cool the plate down. Unlike stage (2), phase (4) looks like a simple cooling process.

Figure 36 is a plot of the norm of the currents induced within the plate. Joule losses thus have the same distribution.

Another meaningful experiment consists in fitting the temperature curve to an exponential function. Setting the magneto-mechanical coupling aside and choosing a particular point, the temperature is driven by:

$$
k \Delta T(t)+s=\rho C_{p} \partial_{t} T(t),
$$

where the single variable is $t$. The solution is of the form $T=A\left(1-e^{\frac{-t}{\tau}}\right)$. During the heating process the real evolution of the temperature is more complex and has no analytical form. But the curve can still be fitted with the previous function. It highlights the small bumps lying on

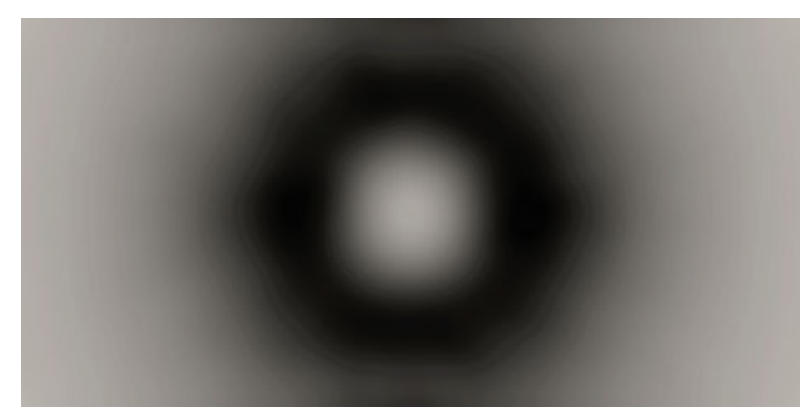

Fig. 36. Distribution of Joule losses within the plate. Eddy currents are located accordingly. 


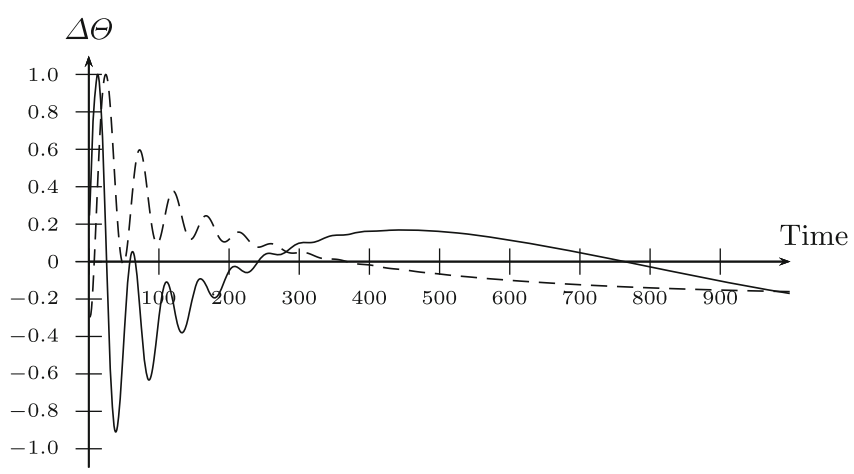

Fig. 37. Solid: plot of the normalized difference between the temperature and the fitted curve Dashed: normalized value of the displacement, $\pi / 2$ phase shifted.

the computed curve (see Fig. 34). One can observe that the difference between the real curve and the fitted one varies according to the instantaneous state of the air gap (see Fig. 37). Because of the time derivative in equation (14), these two curves are $\pi / 2$ phase shifted.

\subsubsection{Comments on the numerical method}

We have deliberately ensured that the characteristic times of the three models are sufficiently different. This greatly facilitates the interpretation of the results.

This example illustrates the power of the projection method. If computational tools are available, this is the basis to the setup of a generic flowchart. Hence a wide scope of use is possible, and complex coupled phenomena can be easily modeled.

\section{Conclusion}

This work presents an innovative method to transfer data from a physical model to another one. The numerical method is of high interest as it enables the use of heterogeneous computational tools. For the first time, the projection methods applied to the multi-physics modeling of systems were successfully tested on a three-physics problem. At the same time, major drawbacks of the coupling methods are overcome: meshes can be numerically adapted to the physical models. Furthermore, computational and memory costs were reduced.

We have firstly presented the projection methods using several dot products. Among this set of tools we have demonstrated that some of them are, depending on the values of interest, most suitable for data transfer. Therefore complex systems can be modeled in different ways: starting from the projection methods available, we have tried to determine the most accurate one. Once defined, experiments on heavily coupled systems have been successfully carried out.

The projections greatly depend on the considered basis function. For each kind of element, two specific dot products can be proposed: the one derived from the $\mathcal{L}^{2}$ subspace and the one derived from the $\mathcal{H}^{1}$ sub-space. The

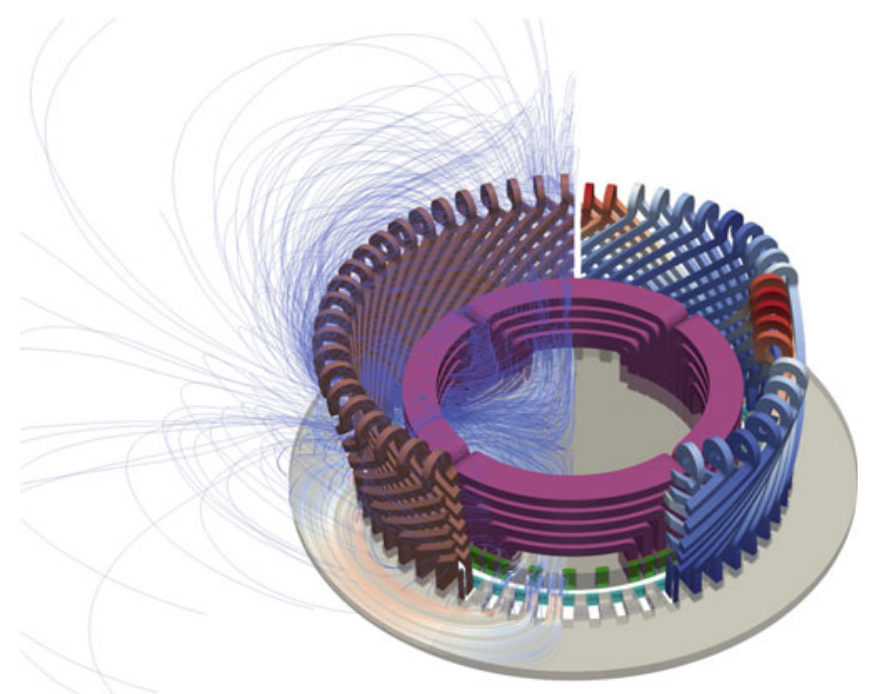

Fig. 38. Magneto-mechanical model of end connections of a 1 GW turbo-alternator used in a nuclear power plant: elastic deformations, present during normal operation, are computed as part of a fatigue prediction study. The magnetic and mechanical models have resort to distinct meshes and are linked by the developed projection method. Left part is the undeformed geometry for which magnetic field lines were added. Right part presents an oversized $\left(10^{5}\right)$ deformation of the windings.

inner product to choose depends on the value of interest. In the case where physical fields are used through the second mesh, results recommend the use of $\mathcal{H}^{1}$ norm for potentials. Nevertheless, the $\mathcal{L}^{2}$ projection method is recommended for a good conservation of the considered value, no matter its physical meaning. The test-cases point out this statement although the accuracy depends on both the solution and the mesh. The efficiency of the mesh intersection computation appeared to be the key point of the method.

Associated with quadratic basis functions and an appropriate reduction of the different computation domains, we have found that force density, Joule losses, temperature and displacement are attractive choices for data transfer. Under these provisos, the overall error added to the mechanical strain is quite acceptable. Three independent test-cases were used for performance comparison.

The efficiency of the proposed method is particularly noticeable when applied to complex problems. Despite their heavily coupled behavior, the two dynamical systems presented in this article were easily modeled. More than a simple computation method, this paper presents a new approach for coupled systems computation. The wide availability of high-quality computer codes is attractive knowing that most of them are open sources. We have attempted to produce a flexible method that - in addition to the reduction of computational cost - enables the use of numerically adapted meshes.

This work aims to present benefits provided by the coupling method using disconnected meshes and projections. It is intended to be used to model industrial applications: the present code is involved in the computation of vibrations within the end connections of large 
turbo-alternators. Figure 38 presents an example of computation: left part is the undeformed geometry and the magnetic field lines while the right part is the elastic deformation (not at scale) of the end connections induced by the magnetic forces. These forces are present during normal operation and are source of aging of the windings. The operator has interest to monitor these deformations in order to predict possible failure, or modifications of the behavior in the case of structural changes.

This particular example involves magnetism, thermal science and applied mechanics. However, the coupling method involving projections can be used in numerous other cases.

\section{References}

1. Z. Ren, B. Ionescu, M. Besbes, A. Razek, IEEE Trans. Magn. 31, 1873 (1995)

2. M. Besbes, Z. Ren, A. Razek, IEEE Trans. Magn. 32, 1058 (1996)

3. A. Bossavit, I.D. Mayergoyz, Computational Electromagnetism: Variational Formulations, Complementarity, Edge Elements (Elsevier Science, Boston, 1998)

4. Z. Ren, IEEE Trans. Magn. 30, 3471 (1994)

5. Z. Ren, A. Razek, IEEE Trans. Magn. 28, 1212 (1992)

6. A. Bossavit, Int. J. Appl. Electromagn. Mater. 2, 333 (1992)

7. A. Bossavit, Eur. J. Mech. B Fluids 10, 474 (1991)

8. A. Bossavit, Int. Compumag Soc. Newsletter 11, 12 (2004)

9. A. Bossavit, Compel 26, 932 (2007)

10. A. Bossavit, IEEE Trans. Magn. 44, 1158 (2008)

11. T. Kovanen, T. Tarhasaari, L. Kettunen, IEEE Trans. Magn. 47, 894 (2011)

12. G. Parent, P. Dular, F. Piriou, A. Abakar, IEEE Trans. Magn. 45, 1132 (2009)
13. P.G. Ciarlet, in Studies in Mathematics and its Applications (Elsevier Science, North-Holland, Amsterdam, 1978)

14. F. Alauzet, M. Mehrenberger, P1-conservative solution interpolation on unstructured triangular meshes. Rapport de recherche RR-6804, INRIA, Rocquencourt, France, 2009

15. O. Devillers, P. Guigue, Technical Report RR-4488, INRIA, 2002

16. N. Nemitz, O. Moreau, Y. Ould-Rouis, Magneto-thermal coupling: A conservative-based method for scalar field projection, in 18th Conference on the Computation of Electromagnetic Fields (Compumag 11), Sydney, Australia, 2011

17. Z. Wang, A.A. Journeaux, T. Henneron, F. Bouillault, N. Nemitz, J.-Y. Roger, J.C. Mipo, F. Piriou, Modelling of magneto-elastic problems on disconnected meshes, in Seventh European Conference on Numerical Methods in Electromagnetism, Marseille, France, 2012

18. B. Flemisch, B.I. Wohlmuth, Numer. Methods Partial Differ. Equ. 20, 374 (2004)

19. B.I. Wohlmuth, SIAM J. Numer. Anal. 38, 989 (1998)

20. B.I. Wohlmuth, Modél. Math. Anal. Numér. 36, 995 (2002)

21. A. Buffa, F. Rapetti, Math. Mod. Methods Appl. Sci. 35, 191 (1999)

22. F.B. Belgacem, Numer. Math. 84, 173 (1999)

23. T. Morisue, IEEE Trans. Magn. 26, 540 (1990)

24. Z. Ren, A. Razek, IEEE Trans. Magn. 26, 1650 (1990)

25. F.C. Moon, Magneto-solid mechanics (Wiley-Interscience Publication, Wiley, New York, 1984)

26. A.A. Journeaux, F. Bouillault, J.-Y. Roger, Magnetomechanical dynamic system modelling using computer code chaining and field projections, in IEEE Conference on Electromagnetic Field Computation, Oita, Japan, 2012 27. K. Yuan, Appl. Sci. Res. 26, 307 (1972)

Open Access This article is distributed under the terms of the Creative Commons Attribution Noncommercial License which permits any noncommercial use, distribution, and reproduction in any medium, provided the original author(s) and source are credited. 